\title{
Antifungal Topical Nanoemulgel Containing Miconazole Nitrate
}

\author{
Anju K P', Shripathy $D^{2}$, Shabaraya $A^{1} ~ R^{3}$ \\ ${ }^{1}$ M.Pharm, Department of Pharmaceutics, Srinivas College of Pharmacy Valachil, Mangalore.574143, \\ Karnataka, India. \\ ${ }^{2}$ Assistant Professor, Department of Pharmaceutics, Srinivas College of Pharmacy, Mangalore, India. \\ ${ }^{3}$ Principal, Department of Pharmaceutics, Srinivas College of Pharmacy, Mangalore, India.
}

Corresponding Author: Anju K P

\begin{abstract}
Nanomulgel have emerged as one of the most interesting topical drug delivery system as it has dual release control system i.e. nanoemulsion and gel. Also the stability of nanoemulsion is increased when it is incorporated in gel. Miconazole nitrate is an antifungal medication topically administered to treat skin infections such as athlete's foot, jock itch and ringworm. The aim of the present research work was to investigate the potential of nanoemulgel in enhancing the topical delivery of hydrophobic drug. MCZ nanoemulsions were prepared using span 80, tween 80, propylene glycol and different conc. of sunflower oil by High pressure homogenization technique. The prepared nanoemulsions were evaluated for $\mathrm{pH}$, drug content, centrifugation, globule size and zeta potential. F2 showed highest drug content $91.26 \%$.The globule size are found to be satisfactory range of nanoemulsion. The drug release kinetics is in the order of F2>F3>F4>F5>F1.And Nanoemulgel is prepared by using Carbopol 934 as gelling agent The release kinetics of nanoemulgel was found to obey zero order kinetics. The nanoemulgel was found to be stable with respect to physical appearance, $\mathrm{pH}$, rheological properties spreadability and drug content at all temperature and conditions for two months. Hence, in the present study it can be concluded that Miconazole Nitrate nanoemulgel formulation is a promising system for the topical drug delivery and also an alternative method to deliver the hydrophobic drugs in water soluble gel bases.
\end{abstract}

Key Words: Hydrophobic drugs, Nanoemulgel, Miconazole nitrate, Topical drug delivery.

\section{INTRODUCTION}

For decades, human skin has provided a unique location for the delivery of a variety of medicines, both systemically and locally. The direct accessibility of the skin as a target organ for diagnostic and therapy is a unique characteristic of dermatological pharmacology.

The use of translucent gels in cosmetics and medicinal preparations has increased within the primary category of semisolid preparations. Gels are a more recent type of dosage form that is made by encasing significant volumes of aqueous or hydroalcoholic liquid in a network of colloidal solid particles. When opposed to an ointment or cream base, they contain a larger aqueous component, which allows for better drug solubility and easy drug migration via a vehicle that is virtually a liquid. In terms of ease of use and patient acceptance, these are far superior. Despite the many benefits of gels, hydrophobic drug delivery is a major limitation. To get around this limitation, nanoemulgels are created and used, allowing even a hydrophobic therapeutic moiety to benefit from gels' unique properties. ${ }^{3,5}$.

Nanoemulgels are dosage formulations that mix gels with nanoemulsions. The main goal of nanoemulgel drug delivery is to distribute 
hydrophobic medicines so that they can benefit from the benefits of gel formulation as well. Because the gelling capacity of these compounds allows the formulation of stable emulsions and creams by decreasing surface and interfacial tension while simultaneously increasing the viscosity of the aqueous phase, there has been a lot of interest in using novel polymers with complex functions as emulsifiers and thickeners in recent years. Nanomulsions are elegant and easy to remove. They can also penetrate the skin quite well. ${ }^{12 \text {, }}$

Miconazole nitrate comes as creams and lotions that may be purchased commercially. The low spreading coefficient, sticky character, and lack of stability are all disadvantages of these formulations. Topical nanoemulgel formulations have been offered as a way to get around these drawbacks. As a result, Nanomulgels have shown to be a great boon in the administration of hydrophobic medicines topically while also offering gel formulation benefits. The aim of the present research work is to design, develop and evaluate miconazole nitrate nanoemulgel for topical fungal disease.

\section{MATERIALS AND METHODS MATERIALS}

Antifungal drug i.e., Miconazole nitrate from yarrow chemicals, Mumbai, india. Carbopol 93 Yarrow chemicals., Sunflower oil from local company. Tween 80; Span 80, Methanol; Hi-Media laboratory Pvt. Ltd, Mumbai, India. Propylene glycol from Loba cheme laboratory., Methyl paraben from SD Fine Chem limited, Mumbai. propyl paraben was purchased from Loba cheme laboratory. Distilled water was used for all experiments. All chemicals were of pharmaceutical grade and used without further modification.

\section{Methodology}

High pressure homogenization method used for the formulation,

There are 3 steps involved,
2. Preparation of gel

3. Incorporation of nanoemulsion into gel

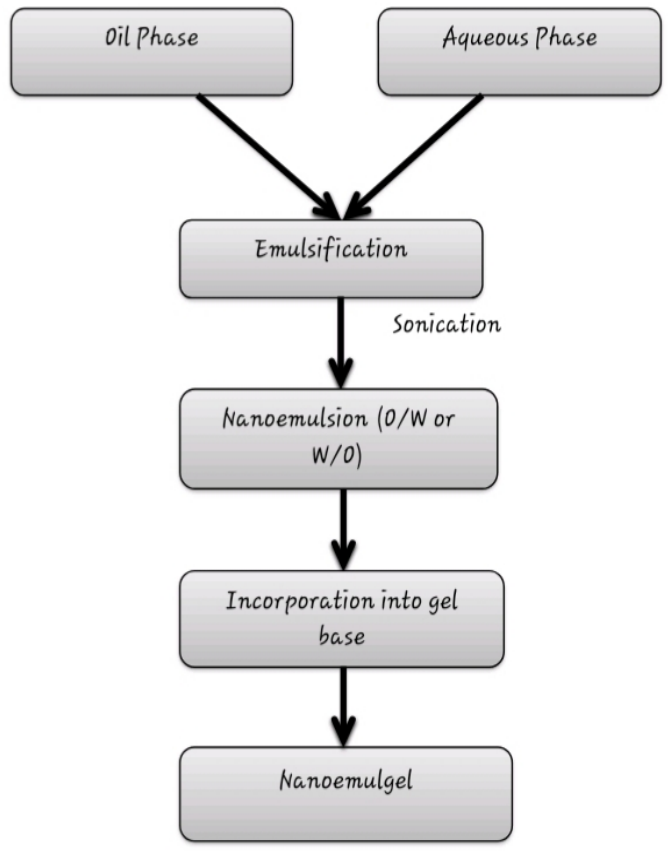

Fig.no 01: Flow chart of preparation

\section{Preparation of nanoemulsion}

High pressure homogenization was used to make a miconazole nitrate nanoemulsion. The nanoemulsion's oil phase was made by dissolving span 80 in sunflower oil, while the aqueous phase was made by dissolving tween 80 in filtered water. The drug was dissolved in methanol, while the methyl and propyl parabens were dissolved in propylene glycol, and both solutions were combined with the oil phase. Both the oily and aqueous phases were heated to $70-80{ }^{\circ} \mathrm{C}$ separately, then the oily phase was introduced to the aqueous phase and homogenised for 1 hour before cooling to room temperature.

Tab no.01: Composition of nanoemulsion

\begin{tabular}{|l|l|l|l|l|l|}
\hline $\begin{array}{l}\text { Ingredients/formulation } \\
\text { code }\end{array}$ & F1 & F2 & F3 & F4 & F5 \\
\hline Miconazole nitrate (w/w) & 0.25 & 0.25 & 0.25 & 0.25 & 0.25 \\
\hline Sunflower oil (v/v) & 5 & 4.5 & 4 & 3.5 & 3 \\
\hline Tween 80 (v/v) & 0.1 & 0.1 & 0.1 & 0.1 & 0.1 \\
\hline Span 80 (v/v) & 0.15 & 0.15 & 0.15 & 0.15 & 0.15 \\
\hline Propylene glycol (v/v) & 4 & 4 & 4 & 4 & 4 \\
\hline Methanol (v/v) & 2 & 2 & 2 & 2 & 2 \\
\hline Methyl paraben (w/w) & 0.01 & 0.01 & 0.01 & 0.01 & 0.01 \\
\hline Propyl paraben (w/w) & 0.05 & 0.05 & 0.05 & 0.05 & 0.05 \\
\hline Water (v/v) & 25 & 25 & 25 & 25 & 25 \\
\hline
\end{tabular}

1. Preparation of nanoemulsion 


\section{Characterization of Nanoemulsion 1. Physical examination ${ }^{35}$}

The visual appearance, phase separation, homogeneity, and consistency of the produced nanoemulsion formulations were all screened.

\section{Centrifugation stability study ${ }^{35}$}

Distilled water was used to dilute nanoemulsions. The nanoemulsions were then centrifuged at $1000 \mathrm{rpm}$ for 15 minutes at $30^{\circ} \mathrm{C}$ to check for changes in homogeneity.

\section{Measurement of $\mathbf{p H}^{35}$}

A digital $\mathrm{pH}$ metre was used to determine the $\mathrm{pH}$ of nanoemulsion formulations. $\mathrm{pH}$ was measured by dissolving $1 \mathrm{~mL}$ of nanoemulsion in $100 \mathrm{~mL}$ of pure water.

\section{Drug content determination ${ }^{55}$}

A UV visible spectroscopic method was used to determine the drug content of a nanoemulsion formulation. Nanoemulsion formulation with methanol yielded a $2 \mathrm{~g} / \mathrm{ml}$ aliquot. At $\lambda$ max, the samples were measured. Three copies of the results were taken.

\section{Globule size determination ${ }^{54}$}

Malvernzeta sizer was used to determine the nanoemulsion's globule size. With the help of a plastic syringe or micropipette, the nanoemulsion (1-1.5 ml) was transferred to a disposable polystyrene cuvette, and the droplet size of the nanoemulsion was determined using a combination of laser doppler velocimetry and phase analysis light scattering (PALS) at a $90^{\circ}$ angle at $25^{\circ} \mathrm{C}$.

\section{Zeta potential ${ }^{54}$}

Zetasizer was used to calculate the zeta potential. Because electrical charges on particles impact the rate of flocculation, the zeta potential is primarily helpful for evaluating flocculation.

\section{In-vitro Drug Release Study}

The drug release tests were conducted using a Franz diffusion cell (effective diffusion area $3.14 \mathrm{~cm} 2$ and cell volume $110 \mathrm{ml}$ ). In a cellophane membrane, nanoemulsion $(5 \mathrm{ml})$ is taken. Between the donor and receptor chambers of the diffusion cell, a cellophane membrane was clamped. To solubilize the drug, the receptor chamber was filled with a 25ML solution of newly produced phosphate buffer (pH 5.5) and methanol (80:20) solution. A magnetic stirrer was used to agitate the receptor chamber. After proper dilutions, the samples were collected at appropriate time intervals and tested for drug content using a UV visible spectrophotometer at $\lambda$-max.

\section{Drug release kinetic studies ${ }^{30}$}

The release data were fitted to zero order, first order, Higuchi model, and korsmeyer's peppas models to evaluate the mechanism of drug release from the topical nanoemulsion.

\section{Preparation of gel}

Preparation of carbopol $934 \mathrm{Gel}^{24}$

The carbopol gel formulations were made by dispersing Carbopol 934 in filtered water while stirring constantly at a moderate speed, and then adjusting the $\mathrm{pH}$ to 6 to 6.5 using Tri ethanol amine (TEA).

To make the MCZ nanoemulgel, nanoemulsion was combined with the gel in a 1:1 ratio with moderate stirring.

Tab no.02: composition of nanoemulgel

\begin{tabular}{|l|l|l|l|l|l|}
\hline Ingredients/ Formulation code & GF1 & GF2 & GF3 & GF4 & GF5 \\
\hline MCZ Nanoemulsion (w/v) & $25 \mathrm{ml}$ & $25 \mathrm{ml}$ & $25 \mathrm{ml}$ & $25 \mathrm{ml}$ & $25 \mathrm{ml}$ \\
\hline Carbopol 934 (w/w) & 1 & 1 & 1 & 1 & 1 \\
\hline Glutaradehyde (v/v) & $0.02 \mathrm{ml}$ & $0.02 \mathrm{ml}$ & $0.02 \mathrm{ml}$ & $0.02 \mathrm{ml}$ & $0.02 \mathrm{ml}$ \\
\hline Triethanolamine (v/v) & $0.05 \mathrm{ml}$ & $0.05 \mathrm{ml}$ & $0.05 \mathrm{ml}$ & $0.05 \mathrm{ml}$ & $0.05 \mathrm{ml}$ \\
\hline Distilled water (qs) (v/v) & $50 \mathrm{ml}$ & $50 \mathrm{ml}$ & $50 \mathrm{ml}$ & $50 \mathrm{ml}$ & $50 \mathrm{ml}$ \\
\hline
\end{tabular}




\section{CHARACTERIZATION OF NANOEMULGEL}

Prepared nanoemulgel of Miconazole Nitrate were evaluated for the following parameters.

\section{Physical Examination ${ }^{30}$}

The colour, homogeneity, consistency, and phase separation of the produced nanoemulgel formulations were visually examined.

\section{Measurement of $\mathbf{p H}^{30}$}

A digital $\mathrm{pH}$ metre was used to determine the $\mathrm{pH}$ of nanoemulgel compositions. $1 \mathrm{~g}$ of gel was dissolved in $100 \mathrm{~mL}$ distilled water, left for 2 hours, and the $\mathrm{pH}$ was measured.

\section{Rheological Study ${ }^{30}$}

A Brookfield Viscometer with spindle 64 was used to determine the viscosity of the prepared batches. The viscosity of the formulation to be determined was added to the beaker. The spindle was lowered perpendicular to the nanoemulgel's centre, taking care not to contact the adapter's bottom, and cycled at a speed of $100 \mathrm{rpm}$.

\section{Spreadability ${ }^{57}$}

Two glass slides $(14 * 5 \mathrm{~cm})$ of identical length were used to test the gel formulation's spreadability. 1gm gel was applied to one of the glass slides, the other slide was placed over it, and weights (125g) were placed on it, and the time it took for the glass slide to slip away from the first glass slide was measured in seconds. Better spreadability is indicated by a shorter interval. Spreadability was calculated by using the formula,

$\mathrm{S}=\mathrm{M} * \mathrm{~L} / \mathrm{T}$

Where, $\mathrm{S}=$ spreadability, $\mathrm{M}=$ Weight tied to upper slide, $\mathrm{L}=$ Length of glass slides, $\mathrm{T}$
$=$ Time taken to separate the slides completely from each other.

\section{Drug Content Determination ${ }^{57}$}

By dissolving $1 \mathrm{~g}$ of nanoemulgel in $100 \mathrm{ml}$ of solvent, the drug concentration in Gellified nanoemulsion was determined (methanol). In a UV/VIS spectrophotometer, absorbance was measured after a sufficient dilution at $\lambda$ max.

\section{In-vitro drug release study ${ }^{56}$}

The drug release tests were conducted using a Franz diffusion cell (effective diffusion area $3.14 \mathrm{~cm} 2$ and cell volume $110 \mathrm{ml}$ ). Nanoemulgel (1g) was placed to the cellophane membrane's surface. Between the donor and receptor chambers of the diffusion cell, a cellophane membrane was clamped. To solubilize the drug, the receptor chamber was filled with a 25ML solution of freshly prepared phosphate buffer ( $\mathrm{pH}$ 5.5) and methanol (80:20) solution. A magnetic stirrer was used to agitate the receptor chamber. After proper dilutions, the samples were collected at appropriate time intervals and tested for drug content using a UV visible spectrophotometer at $\lambda$-max.

\section{Drug release kinetic studies ${ }^{30}$}

The release data was fitted to Zero order, First order kinetics, Higuchi's, and Korsmeyers peppa's equations to investigate the mechanism of drug release from the topical gel.

\section{Accelerated stability studies ${ }^{35,58}$}

The best formulations were submitted to two months of stability testing at $4020 \mathrm{C}$ and $75 \% \mathrm{RH}$. At two-month intervals, parameters such as appearance, drug content, phase separation, and in-vitro release were evaluated. 


\section{RESULTS \& DISCUSSION}

Preformulation studies of miconazole nitrate

\begin{tabular}{|c|c|c|c|}
\hline \multicolumn{2}{|l|}{ Properties } & Reported & Observed \\
\hline \multicolumn{2}{|c|}{ Appearance } & White crystalline powder & White crystalline powder \\
\hline \multicolumn{2}{|l|}{ Odour } & Odourless & Odourless \\
\hline \multicolumn{2}{|c|}{ Melting point $\left({ }^{0} \mathrm{C}\right)$} & $179-182^{\circ} \mathrm{C}$ & $179^{\circ} \mathrm{C} \pm 1.52$ \\
\hline \multirow[t]{3}{*}{ Solubility } & Methanol & $13 \mathrm{mg} / \mathrm{ml}$ & $10 \mathrm{mg} / \mathrm{ml}$ \\
\hline & PBS pH 5.5 & $0.3 \mathrm{mg} / \mathrm{ml}$ & $0.1 \mathrm{mg} / \mathrm{ml}$ \\
\hline & Propylene glycol & $43 \mathrm{mg} / \mathrm{ml}$ & $40 \mathrm{mg} / \mathrm{ml}$ \\
\hline \multicolumn{2}{|c|}{ Identification (UV) } & $272 \mathrm{~nm}$ & $272 \mathrm{~nm}$ \\
\hline
\end{tabular}

\section{Spectrum measurement}

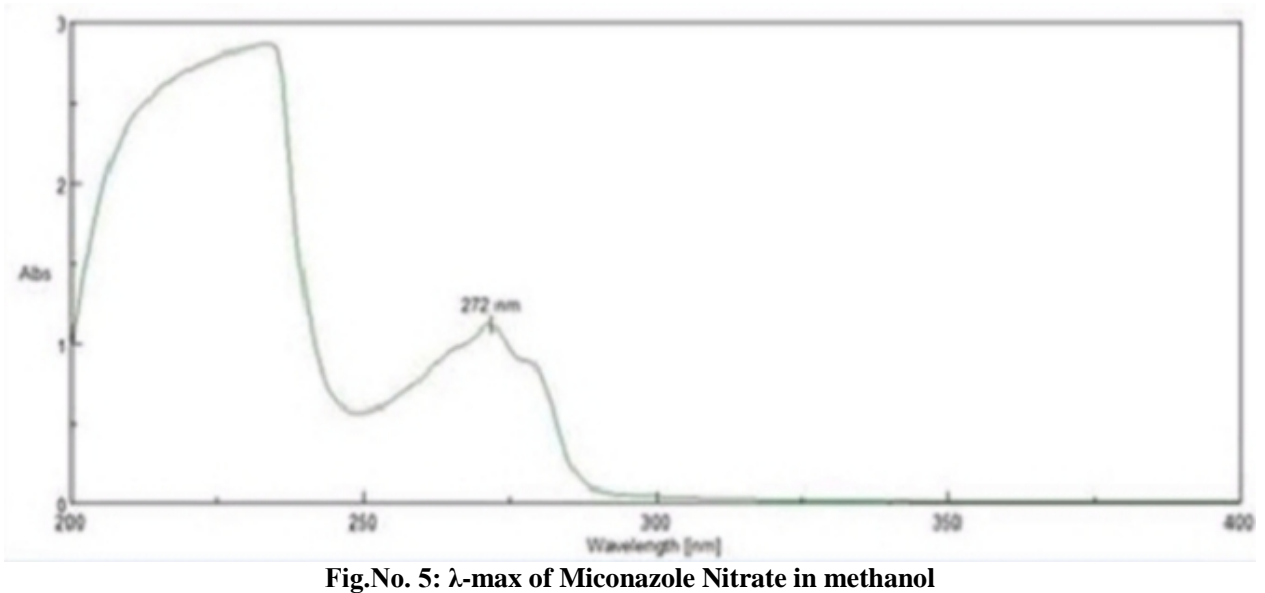

Prior to that, preformulation experiments for the medication were conducted, followed by formulation production and assessment. The following are the outcomes of the following experiment.

\section{Organoleptic characteristics:}

MCZ's organoleptic characteristics, such as general description, colour, smell, and taste, were studied. MCZ was discovered to be a white crystalline powder that is somewhat bitter, odourless, and falls within the published literature limitations. Table No.05 displays the results observed.

\section{Melting point:}

Miconazole Nitrate has a melting point of $1790^{\circ} \mathrm{C}$, which is within the pharmacopoeia's range of $179-1820^{\circ} \mathrm{C}$ with decomposition, confirming purity of the medicinal sample. Table No. 05 shows the result observed.

\section{Solubility:}

Miconazole Nitrate is soluble in methanol, propylene glycol, and phosphate buffer, according to the Indian pharmacopoeia's solubility profile. Table No. 05 shows the data obtained.

\section{Spectrum measurement:}

Between 200 and $400 \mathrm{~nm}$, the absorption spectra of pure Miconazole Nitrate was scanned. In methanol, the $\lambda$ max of pure MCZ was determined to be 272 nm. Figure No. 05 depicts the results achieved.

\section{Standard calibration curve of Miconazole} Nitrate

Tab.No.6: Standard calibration curve of MCZ

\begin{tabular}{|l|l|l|}
\hline $\begin{array}{l}\text { SI } \\
\text { No. }\end{array}$ & $\begin{array}{l}\text { Concentration of Miconazole Nitrate } \\
(\boldsymbol{\mu} \mathbf{g} / \mathbf{m l})\end{array}$ & Absorbance \\
\hline 01. & 0 & $0 \pm 0$ \\
\hline 02. & 5 & $0.05 \pm 0.01$ \\
\hline 03. & 10 & $0.11 \pm 0.01$ \\
\hline 04. & 15 & $0.16 \pm 0.01$ \\
\hline 05. & 20 & $0.21 \pm 0.02$ \\
\hline 06. & 25 & $0.25 \pm 0.01$ \\
\hline 07. & 30 & $0.3 \pm 0.05$ \\
\hline
\end{tabular}




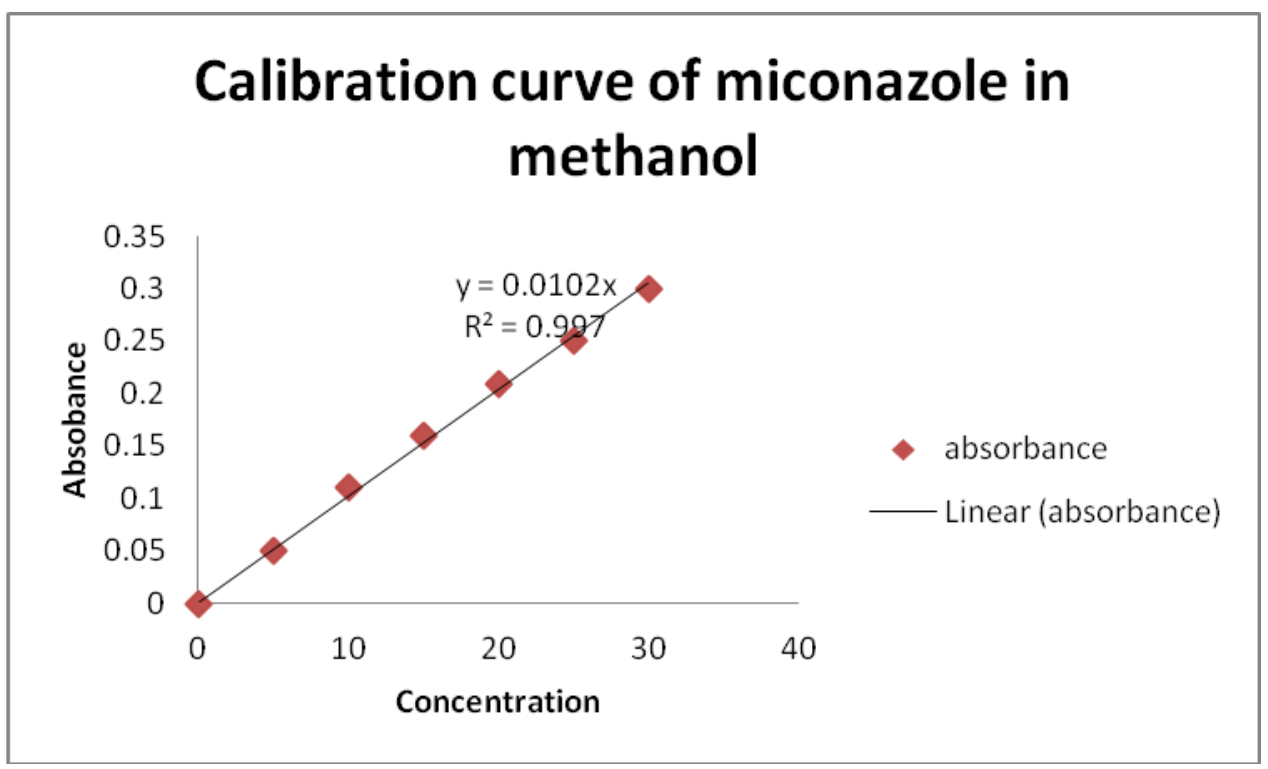

Fig.No.05: Standard calibration curve of Miconazole Nitrate

Miconazole Nitrate calibration curve was obtained at a wavelength of $272 \mathrm{~nm}$ in the concentration range of $5-30 \mathrm{~g} / \mathrm{ml}$. It exhibits high linearity, as illustrated in fig. 06, with a regression coefficient of 0.997 (r2 value).

\section{DRUG - POLYMER COMPATABILITY STUDIES}

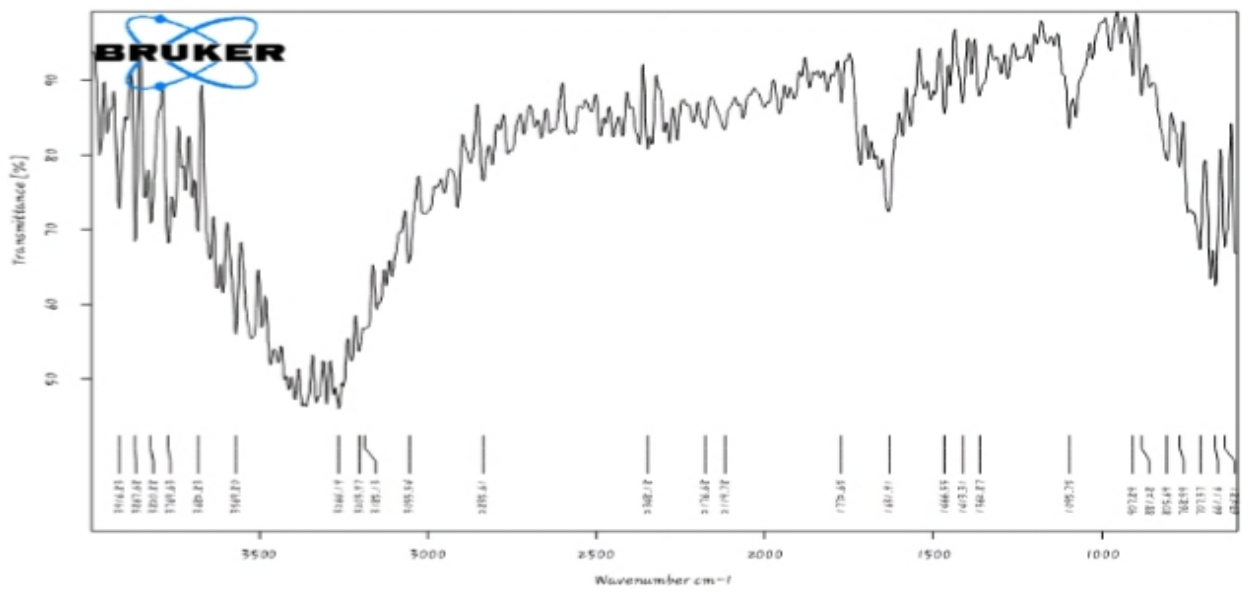

Fig.No.06: FT-IR spectrum of Miconazole Nitrate

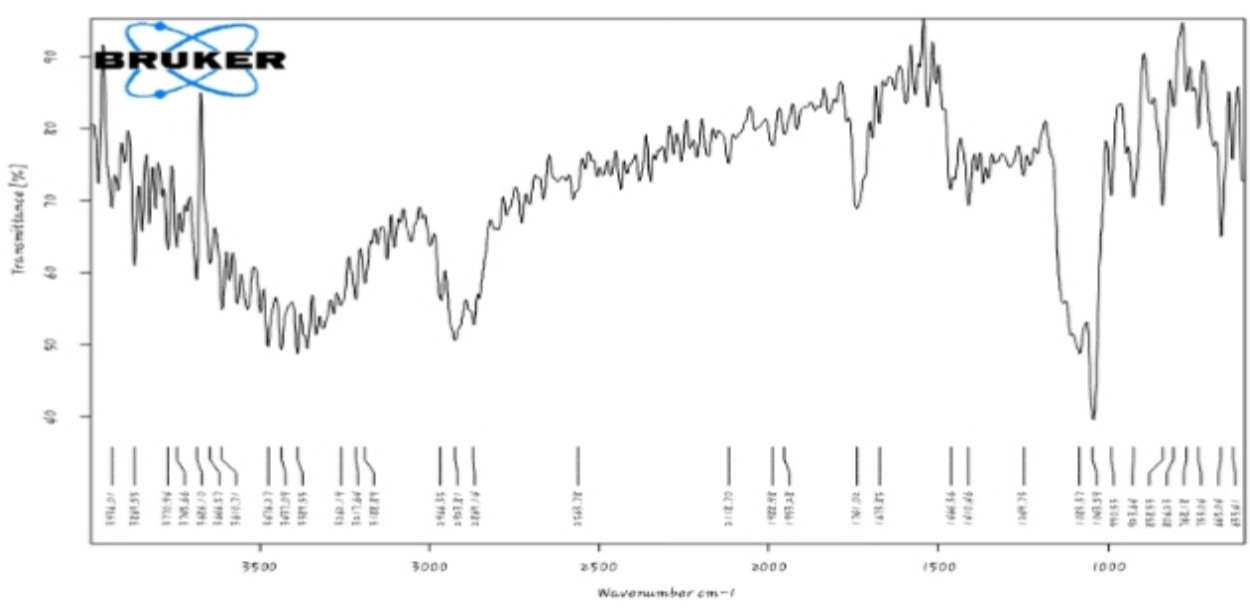

Fig.No.07: FT-IR spectrum of MCZ + POLYMERS 
Tab No.07 : Comparison of FT - IR spectra of MCZ \& Polymers

\begin{tabular}{|l|l|l|l|l|}
\hline SI No & Functional Group & \multirow{2}{*}{ Reported Frequency $\left(\mathbf{c m}^{-\mathbf{1}} \mathbf{)}\right.$} & \multicolumn{3}{|l|}{ Observed frequency } \\
\cline { 3 - 5 } & & & Drug & Drug+Polymers \\
\hline 1 & C=N & 1640 & 1631.91 & 1464.36 \\
\hline 2 & C-H(aliphatic) & 2960 & 2835.61 & 2923.81 \\
\hline 3 & C-H(aromatic) & 3048 & 3055.54 & 2966.35 \\
\hline 4 & C=O & 1635 & 1631.91 & 1464.36 \\
\hline
\end{tabular}

The IR spectra of the drug-polymer combinations and optimal formulation were compared to the standard spectrum of the pure medication Miconazole Nitrate, and the distinctive peaks associated with particular functional groups and the bonds of the molecules were recorded in table no.07.

The peak ranges from $\mathrm{C}=\mathrm{N} 1631.91$

to $1464.36 \mathrm{~cm}^{-1}, \mathrm{C}-\mathrm{H}$ aliphatic 2835.61 to 2923.8cm $\mathrm{cm}^{-1}, \mathrm{C}-\mathrm{H} \quad$ aromatic $3055.54-$ $2966.35 \mathrm{~cm}^{-1}, \mathrm{C}=\mathrm{O} 1631.91$ to $1464.36 \mathrm{~cm}^{-1}$.

The ranges of peak values were found to be same, suggesting that Miconazole Nitrate did not interact with various polymers, indicating the drug's stability in the formulation.

\section{PSEUDO TERNARY PHASE STUDY}

Tab No.08: Pseudo ternary phase studies

\begin{tabular}{|l|l|l|l|l|}
\hline SI. No & ml of Oil & Surfactant mixture(ml) & Water(ml) & Type of emulsion \\
\hline 1 & 1 & 1 & 0 & No Emulsion \\
\hline 2 & 1 & 1 & 1 & Gel \\
\hline 3 & 1 & 7 & 2 & Gel \\
\hline 4 & 1 & 6 & 3 & Nanoemulsion \\
\hline 5 & 1 & 5 & 4 & Nanoemulsion \\
\hline 6 & 1 & 4 & 5 & Emulsion \\
\hline 7 & 1 & 3 & 6 & Gel \\
\hline 8 & 1 & 2 & 7 & Emulsion \\
\hline 9 & 1 & 1 & 8 & Emulsion \\
\hline
\end{tabular}

From the pseudo ternary phase nanoemulsion formation region. The region system, The S/Co S Mixture ratio is found is noted in Tab no.08. out as 3:2, indicated that the best

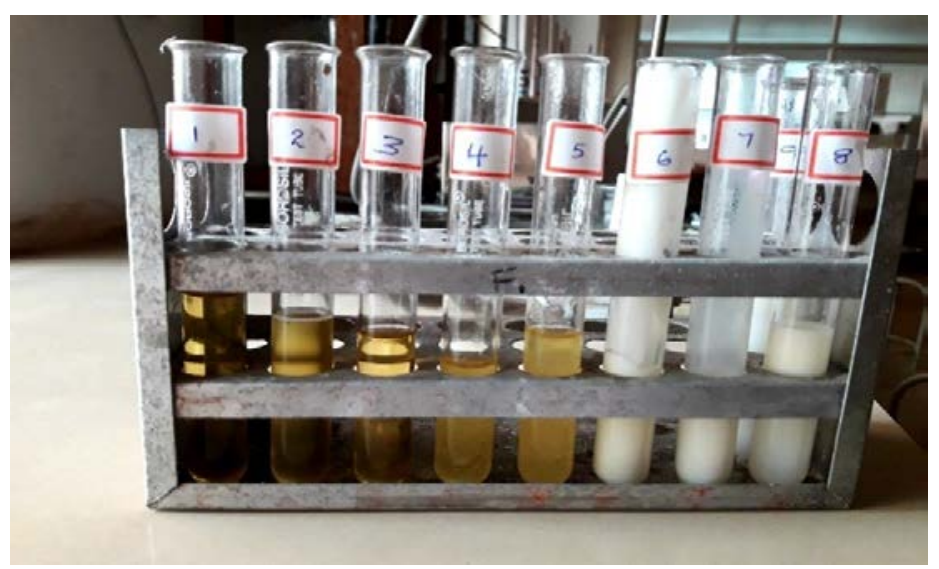

Fig no.08 : Different concentrations of Prepared S/Co-S mixtures

\section{EVALUATION OF NANOEMULSION}

\section{Physical examination}

Tab.No.9: Physical examination of various formulations of nanoemulsion

\begin{tabular}{|l|l|l|l|l|}
\hline Formulation code & Appearance & Phase Separation & Homogenity & Consistency \\
\hline F1 & Milky white & None & Good & Good \\
\hline F2 & Milky white & None & Excellent & Excellent \\
\hline F3 & Milky white & None & Good & Excellent \\
\hline F4 & Milky white & None & Good & Good \\
\hline F5 & Milky white & None & Excellent & Good \\
\hline
\end{tabular}




\section{Physical examination}

Miconazole Nitrate emulsions were produced in a milky white colour with a smooth, homogeneous appearance and outstanding consistency. The formulations showed no signs of phase separation. The outcomes were shown in table No.9. Figure 09 depicts the prepared nanoemulsion.

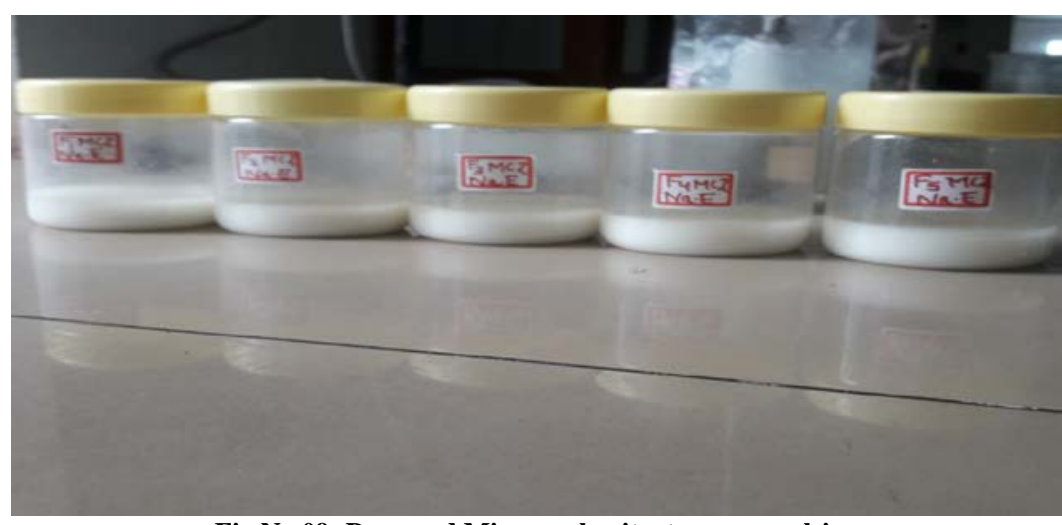

Fig.No.09: Prepared Miconazole nitrate nanoemulsion

\section{Centrifugation stability, $\mathrm{pH}$, Drug content}

Tab.No.10: Centrifugation stability, pH \& Drug content of various formulations of nanoemulsion

\begin{tabular}{|l|l|l|l|}
\hline Formulation code & Centrifugation study & pH & Drug content \% \\
\hline F1 & No phase separation & $6.30 \pm 0.01$ & $79.13 \pm 0.39$ \\
\hline F2 & No phase separation & $6.27 \pm 0.01$ & $91.26 \pm 0.02$ \\
\hline F3 & No phase separation & $6.24 \pm 0.01$ & $88.55 \pm 0.25$ \\
\hline F4 & No phase separation & $6.13 \pm 0.01$ & $86.12 \pm 0.12$ \\
\hline F5 & No phase separation & $6.07 \pm 0.01$ & $81.46 \pm 0.16$ \\
\hline
\end{tabular}

There was no phase separation, indicating that all of the produced nanoemulsions were stable. All formulations had $\mathrm{pH}$ values ranging from 6.07 to 6.30 , which are deemed suitable for avoiding skin irritation when applied to the skin. The drug content of nanoemulsions was determined by spectrophotometry at $272 \mathrm{~nm}$, with drug concentrations ranging from 79.13 to 91.26 percent. The highest drug content was found with F2 (91.26 \%). The results were shown in the table No.10.
Tab no.,11 : Globule size determination of various formulations

\begin{tabular}{|l|l|}
\hline Formulation code & Globule size(d.nm) \\
\hline F1 & 26.82 \\
\hline F2 & 7.9 \\
\hline F3 & 41.6 \\
\hline F4 & 9.4 \\
\hline F5 & 27.72 \\
\hline
\end{tabular}

The mean globule size of nanoemulsion F2 was determined to be 7.9d.nm, which is within the literature limits, demonstrating nanoemulsion homogeneity. Table No.11 and Figure No.11-15 show the results.

\section{Globule size determination}

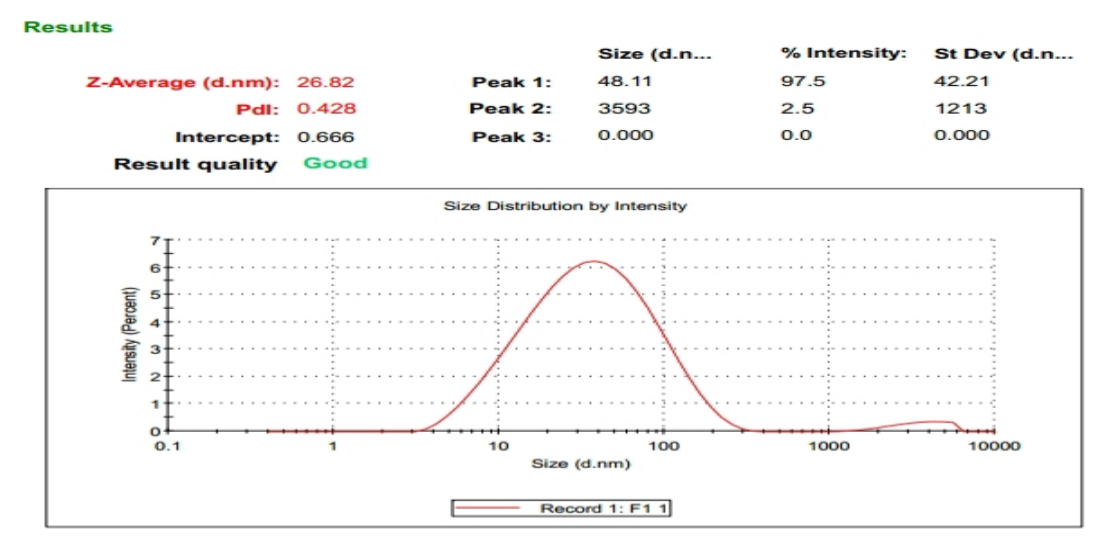

Fig no.11 : Globule size distribution of Nanoemulsion F1 
Anju K P et.al. Antifungal topical nanoemulgel containing miconazole nitrate.

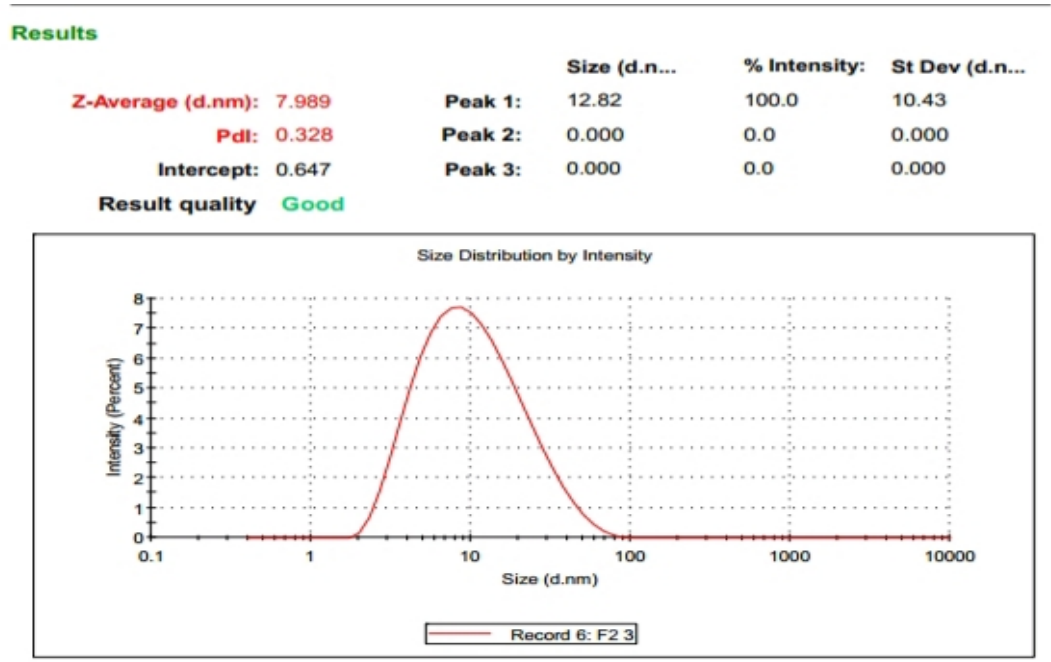

Fig no.12 : Globule size distribution of Nanoemulsion F2

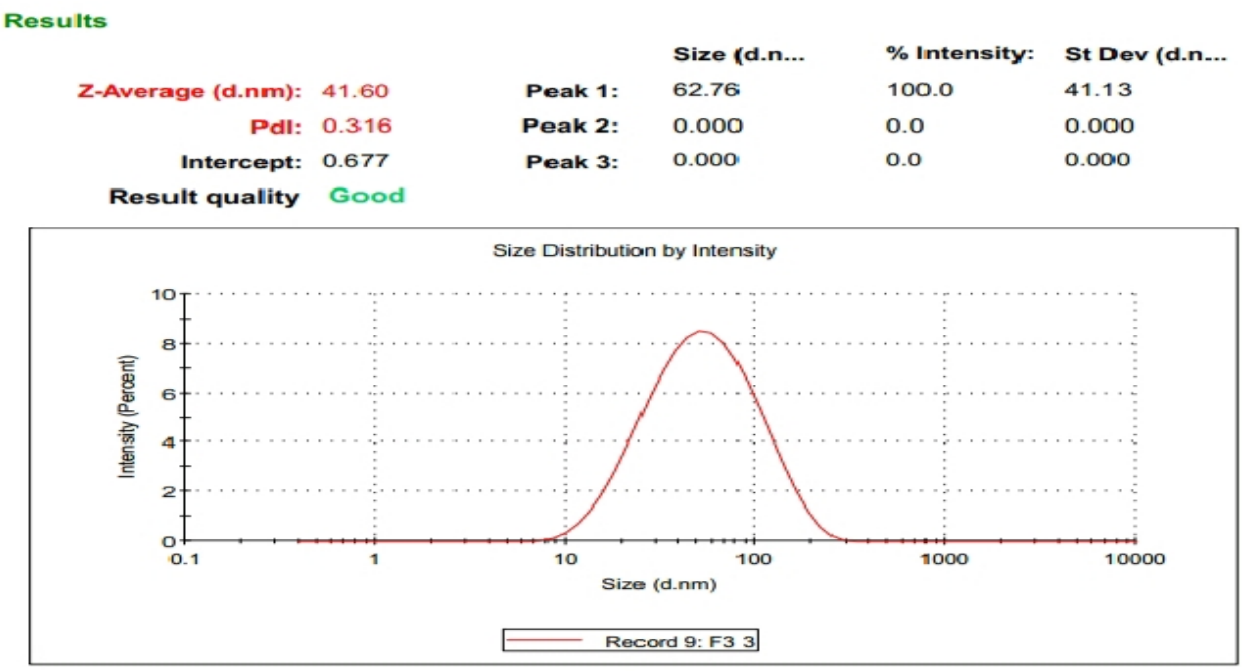

Fig no.13 : Globule size distribution of Nanoemulsion F3

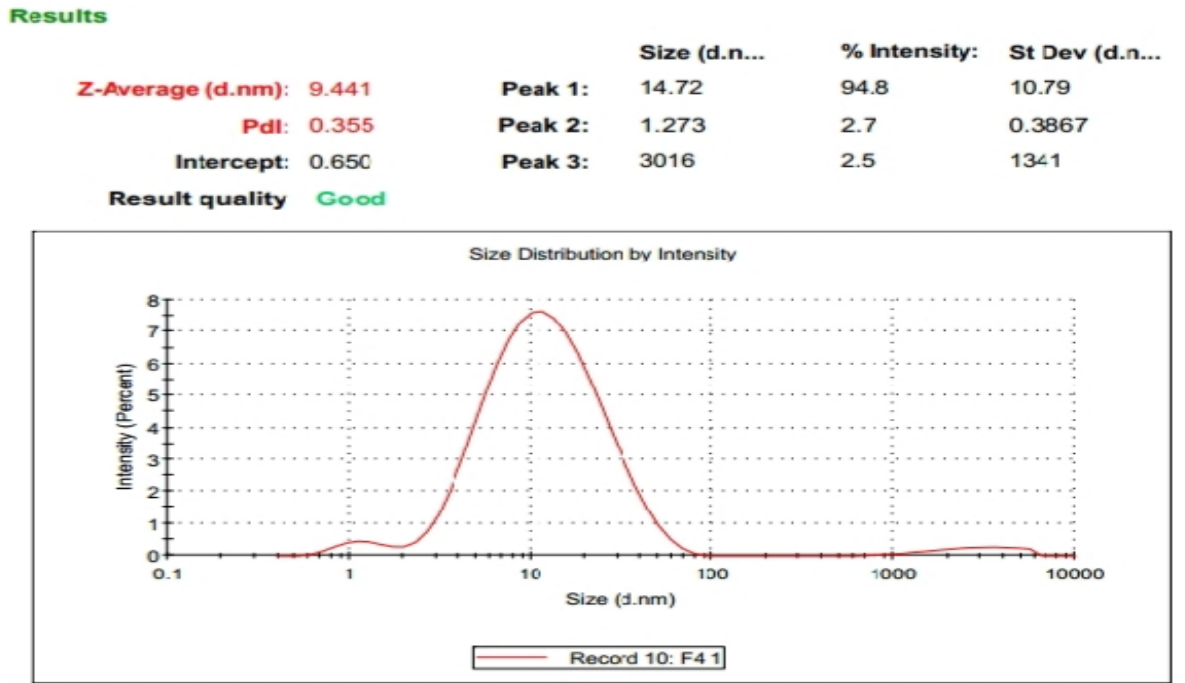

Fig no.14 : Globule size distribution of Nanoemulsion F4 


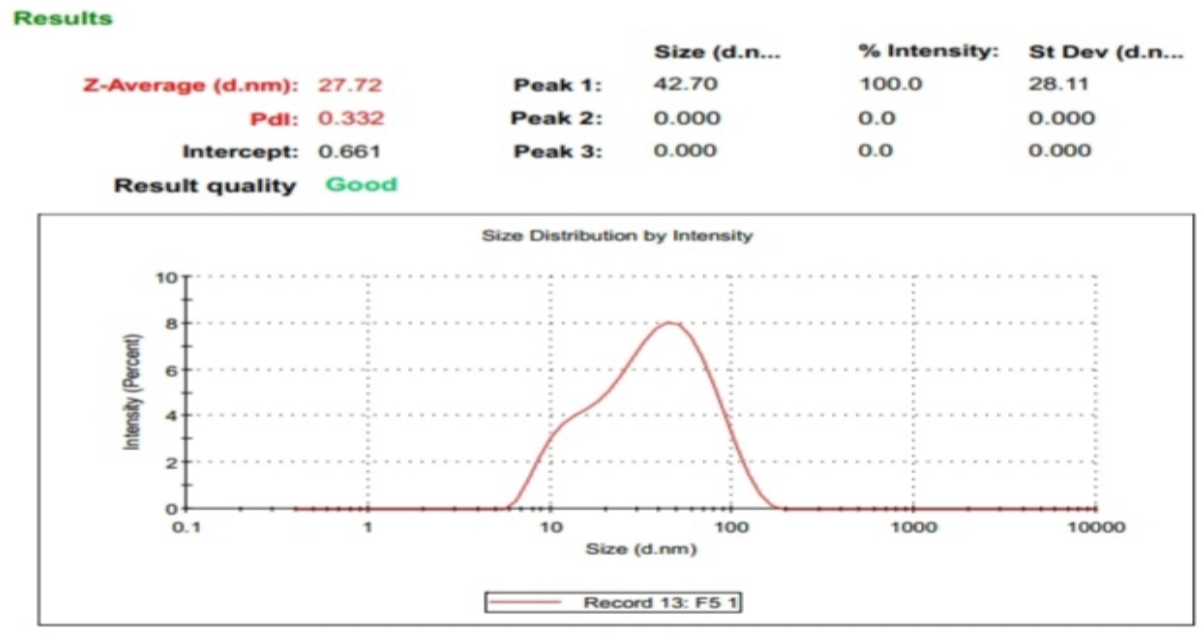

Fig no.15 : Globule size distribution of Nanoemulsion F5

\section{Zeta potential of MCZ Nanoemulsion}

Tab.No.12: Zeta potential of MCZ nanoemulsion F2

\begin{tabular}{|l|l|}
\hline Formulation code & Zetapotential \\
\hline F2 & -32.4 \\
\hline
\end{tabular}

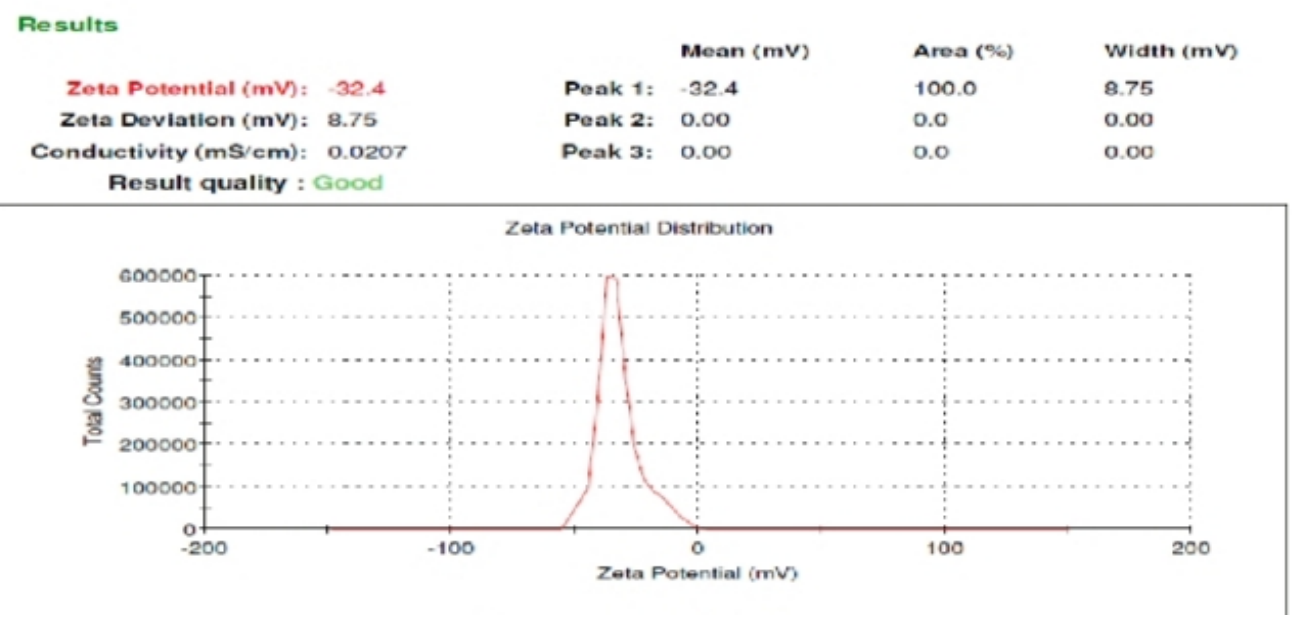

Fig.No.16: Zeta potential graph

Formulation F2's zeta potential was determined to be $-32.4 \mathrm{mV}$. The negative zeta potential means that the nanoemulsion droplets have no charge and that the system is stable since there is no flocculation.

\section{IN-VITRO DRUG RELEASE STUDY}

Tab no.13: In-vitro drug release study of nanoemulsion

\begin{tabular}{|l|l|l|l|l|l|}
\hline \multirow{2}{*}{ Time in hr } & \multicolumn{5}{l}{ Percentage Cumulative Drug Release* } \\
\cline { 2 - 6 } & F1 & F2 & F3 & F4 & F5 \\
\hline 0 & $0 \pm 0$ & $0 \pm 0$ & $0 \pm 0$ & $0 \pm 0$ & $0 \pm 0$ \\
\hline 1 & $3.03 \pm 0.015$ & $6.01 \pm 0.02$ & $8.01 \pm 0.02$ & $4.04 \pm 0.01$ & $7.95 \pm 0.05$ \\
\hline 2 & $4.8 \pm 0.02$ & $8.3 \pm 0.04$ & $10.1 \pm 0.03$ & $4.89 \pm 0.04$ & $11.93 \pm 0.015$ \\
\hline 3 & $6.58 \pm 0.01$ & $10.02 \pm 0.04$ & $13.5 \pm 0.02$ & $5.9 \pm 0.02$ & $14.32 \pm 0.02$ \\
\hline 4 & $8.4 \pm 0.05$ & $12.5 \pm 0.02$ & $17.9 \pm 0.01$ & $7.4 \pm 0.02$ & $18.27 \pm 0.03$ \\
\hline 6 & $13.24 \pm 0.03$ & $16.89 \pm 0.01$ & $23.46 \pm 0.016$ & $13.63 \pm 0.01$ & $24.8 \pm 0.03$ \\
\hline 7 & $17.05 \pm 0.01$ & $21.5 \pm 0.05$ & $29.54 \pm 0.02$ & $19.4 \pm 0.015$ & $26.25 \pm 0.05$ \\
\hline 8 & $20.95 \pm 0.02$ & $28.07 \pm 0.016$ & $32.01 \pm 0.04$ & $23.3 \pm 0.02$ & $34.95 \pm 0.02$ \\
\hline 12 & $36.2 \pm 0.017$ & $42.01 \pm 0.02$ & $46.8 \pm 0.03$ & $40.08 \pm 0.04$ & $48.1 \pm 0.04$ \\
\hline 24 & $72.5 \pm 0.04$ & $92.3 \pm 0.01$ & $90.6 \pm 0.02$ & $88.2 \pm 0.01$ & $88.91 \pm 0.01$ \\
\hline
\end{tabular}




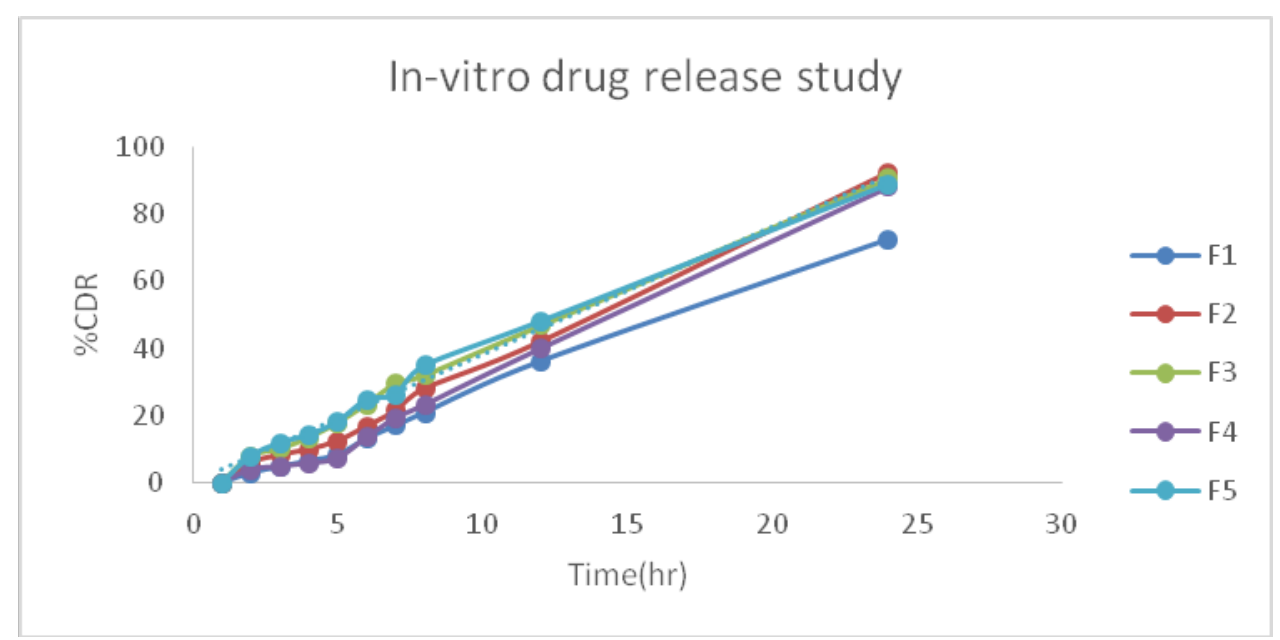

Fig No.17 : In-vitro drug release of various formulations

The drug can be seen being released from all nanoemulsion formulations, and the nanoemulsion formulations may be ordered in the following order: F2 $>$ F3 $>$ F4 $>$ F5 $>$ F1 Tab no. 13 and Fig no. 17 illustrate the results.

\section{DRUG RELEASE KINETIC STUDIES}

Tab no.14: Drug release kinetics of F1

\begin{tabular}{|l|l|l|l|l|l|l|}
\hline Time & $\begin{array}{l}\text { Log } \\
\text { time }\end{array}$ & $\begin{array}{l}\text { Square root of } \\
\text { time }\end{array}$ & $\begin{array}{l}\text { \%cumulative release } \\
\text { of F1 }\end{array}$ & $\begin{array}{l}\text { Log \% cumulative } \\
\text { release of F1 }\end{array}$ & $\begin{array}{l}\text { \%Cumulative } \\
\text { remaining }\end{array}$ & $\begin{array}{l}\text { Log cumulative } \\
\text { remaining }\end{array}$ \\
\hline 0 & 0 & 1 & 0 & 0 & 100 & 2 \\
\hline 1 & 0.30103 & 1.414214 & 3.03 & 0.481443 & 96.97 & 1.986637 \\
\hline 2 & 0.477121 & 1.732051 & 4.8 & 0.681241 & 95.2 & 1.978637 \\
\hline 3 & 0.60206 & 2 & 6.58 & 0.818226 & 93.42 & 1.97044 \\
\hline 4 & 0.69897 & 2.236068 & 8.4 & 0.924279 & 91.6 & 1.961895 \\
\hline 6 & 0.778151 & 2.44949 & 13.24 & 1.121888 & 86.76 & 1.93832 \\
\hline 7 & 0.845098 & 2.645751 & 17.05 & 1.231724 & 82.95 & 1.918816 \\
\hline 8 & 0.90309 & 2.828427 & 20.95 & 1.321184 & 79.05 & 1.897902 \\
\hline 12 & 1.079181 & 3.464102 & 36.2 & 1.558709 & 63.8 & 1.804821 \\
\hline 24 & 1.380211 & 4.898979 & 72.5 & 1.860338 & 27.5 & 1.439333 \\
\hline
\end{tabular}

Tab No.15: Drug release kinetics of F2

\begin{tabular}{|l|l|l|l|l|l|l|}
\hline Time & $\begin{array}{l}\text { Log } \\
\text { time }\end{array}$ & $\begin{array}{l}\text { Square root of } \\
\text { time }\end{array}$ & $\begin{array}{l}\text { \%Cumulative release } \\
\text { of F2 }\end{array}$ & $\begin{array}{l}\text { Log \% cumulative } \\
\text { release of F2 }\end{array}$ & $\begin{array}{l}\text { \%Cumulative } \\
\text { remaining }\end{array}$ & $\begin{array}{l}\text { Log cumulative } \\
\text { remaining }\end{array}$ \\
\hline 0 & 0 & 1 & 0 & 0 & 100 & 2 \\
\hline 1 & 0.30103 & 1.414214 & 6.01 & 0.778874 & 93.99 & 1.973082 \\
\hline 2 & 0.477121 & 1.732051 & 8.3 & 0.919078 & 91.7 & 1.962369 \\
\hline 3 & 0.60206 & 2 & 10.02 & 1.000868 & 89.98 & 1.954146 \\
\hline 4 & 0.69897 & 2.236068 & 12.5 & 1.09691 & 87.5 & 1.942008 \\
\hline 6 & 0.778151 & 2.44949 & 16.89 & 1.22763 & 83.11 & 1.919653 \\
\hline 7 & 0.845098 & 2.645751 & 21.5 & 1.332438 & 78.5 & 1.89487 \\
\hline 8 & 0.90309 & 2.828427 & 28.07 & 1.448242 & 71.93 & 1.85691 \\
\hline 12 & 1.079181 & 3.464102 & 42.01 & 1.623353 & 57.99 & 1.763353 \\
\hline 24 & 1.380211 & 4.898979 & 92.3 & 1.965202 & 7.7 & 0.886491 \\
\hline
\end{tabular}

TabNo.16: Drug release kinetics of F3

\begin{tabular}{|l|l|l|l|l|l|l|}
\hline Time & $\begin{array}{l}\text { Log } \\
\text { time }\end{array}$ & $\begin{array}{l}\text { Square root of } \\
\text { time }\end{array}$ & $\begin{array}{l}\text { \%Cumulative release } \\
\text { of F3 }\end{array}$ & $\begin{array}{l}\text { Log \% cumulative } \\
\text { release of F3 }\end{array}$ & $\begin{array}{l}\text { \%Cumulative } \\
\text { remaining }\end{array}$ & $\begin{array}{l}\text { Log cumulative } \\
\text { remaining }\end{array}$ \\
\hline 0 & 0 & 1 & 0 & 0 & 100 & 2 \\
\hline 1 & 0.30103 & 1.414214 & 8.01 & 0.903633 & 91.99 & 1.963741 \\
\hline 2 & 0.477121 & 1.732051 & 10.1 & 1.004321 & 89.9 & 1.95376 \\
\hline 3 & 0.60206 & 2 & 13.5 & 1.130334 & 86.5 & 1.937016 \\
\hline 4 & 0.69897 & 2.236068 & 17.9 & 1.252853 & 82.1 & 1.914343 \\
\hline 6 & 0.778151 & 2.44949 & 23.46 & 1.370328 & 76.54 & 1.883888 \\
\hline 7 & 0.845098 & 2.645751 & 29.54 & 1.47041 & 70.46 & 1.847943 \\
\hline 8 & 0.90309 & 2.828427 & 32.01 & 1.505286 & 67.99 & 1.832445 \\
\hline 12 & 1.079181 & 3.464102 & 46.8 & 1.670246 & 53.2 & 1.725912 \\
\hline 24 & 1.380211 & 4.898979 & 90.6 & 1.957128 & 9.4 & 0.973128 \\
\hline
\end{tabular}


Tab No.17: Drug release kinetics of F4

\begin{tabular}{|l|l|l|l|l|l|l|}
\hline Time & $\begin{array}{l}\text { Log } \\
\text { time }\end{array}$ & $\begin{array}{l}\text { Square root of } \\
\text { time }\end{array}$ & $\begin{array}{l}\text { \% Cumulative } \\
\text { release of F4 }\end{array}$ & $\begin{array}{l}\text { Log \% cumulative } \\
\text { release of F4 }\end{array}$ & $\begin{array}{l}\text { \%Cumulative } \\
\text { remaining }\end{array}$ & $\begin{array}{l}\text { Log cumulative } \\
\text { remaining }\end{array}$ \\
\hline 0 & 0 & 1 & 0 & 0 & 100 & 2 \\
\hline 1 & 0.30103 & 1.414214 & 4.04 & 0.606381 & 95.96 & 1.98209 \\
\hline 2 & 0.477121 & 1.732051 & 4.89 & 0.689309 & 95.11 & 1.978226 \\
\hline 3 & 0.60206 & 2 & 5.9 & 0.770852 & 94.1 & 1.97359 \\
\hline 4 & 0.69897 & 2.236068 & 7.4 & 0.869232 & 92.6 & 1.966611 \\
\hline 6 & 0.778151 & 2.44949 & 13.63 & 1.134496 & 86.37 & 1.936363 \\
\hline 7 & 0.845098 & 2.645751 & 19.4 & 1.287802 & 80.6 & 1.906335 \\
\hline 8 & 0.90309 & 2.828427 & 23.3 & 1.367356 & 76.7 & 1.884795 \\
\hline 12 & 1.079181 & 3.464102 & 40.08 & 1.602928 & 59.92 & 1.777572 \\
\hline 24 & 1.380211 & 4.898979 & 88.2 & 1.945469 & 11.8 & 1.071882 \\
\hline
\end{tabular}

Tab No.18: Drug release kinetics of F5

\begin{tabular}{|l|l|l|l|l|l|l|}
\hline Time & $\begin{array}{l}\text { Log } \\
\text { time }\end{array}$ & $\begin{array}{l}\text { Square root of } \\
\text { time }\end{array}$ & $\begin{array}{l}\text { \%Cumulative release } \\
\text { of F5 }\end{array}$ & $\begin{array}{l}\text { Log \% cumulative } \\
\text { release of F5 }\end{array}$ & $\begin{array}{l}\text { \%Cumulative } \\
\text { remaining }\end{array}$ & $\begin{array}{l}\text { Log cumulative } \\
\text { remaining }\end{array}$ \\
\hline 0 & 0 & 1 & 0 & 0 & 100 & 2 \\
\hline 1 & 0.30103 & 1.414214 & 7.95 & 0.900367 & 92.05 & 1.964024 \\
\hline 2 & 0.477121 & 1.732051 & 11.93 & 1.07664 & 88.07 & 1.944828 \\
\hline 3 & 0.60206 & 2 & 14.32 & 1.155943 & 85.68 & 1.932879 \\
\hline 4 & 0.69897 & 2.236068 & 18.27 & 1.261739 & 81.73 & 1.912381 \\
\hline 6 & 0.778151 & 2.44949 & 24.8 & 1.394452 & 75.2 & 1.876218 \\
\hline 7 & 0.845098 & 2.645751 & 26.25 & 1.419129 & 73.75 & 1.867762 \\
\hline 8 & 0.90309 & 2.828427 & 34.95 & 1.543447 & 65.05 & 1.813247 \\
\hline 12 & 1.079181 & 3.464102 & 48.1 & 1.682145 & 51.9 & 1.715167 \\
\hline 24 & 1.380211 & 4.898979 & 88.91 & 1.948951 & 11.09 & 1.044932 \\
\hline
\end{tabular}

\section{Zero order}

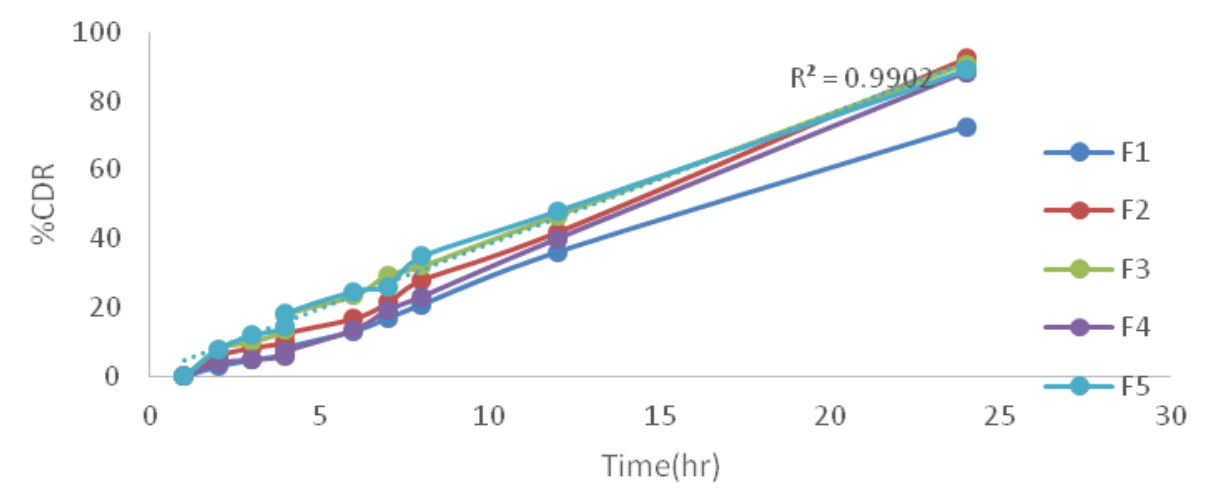

Fig No.18: Plot of \%CDR v/s Time

\section{First order}

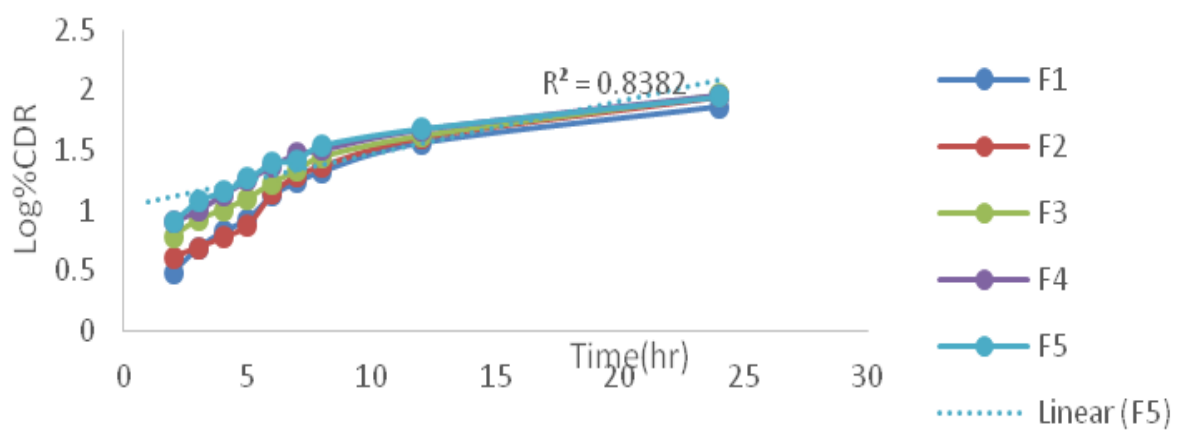

Fig No.19: Plot of log percentage CDR v/s Time 


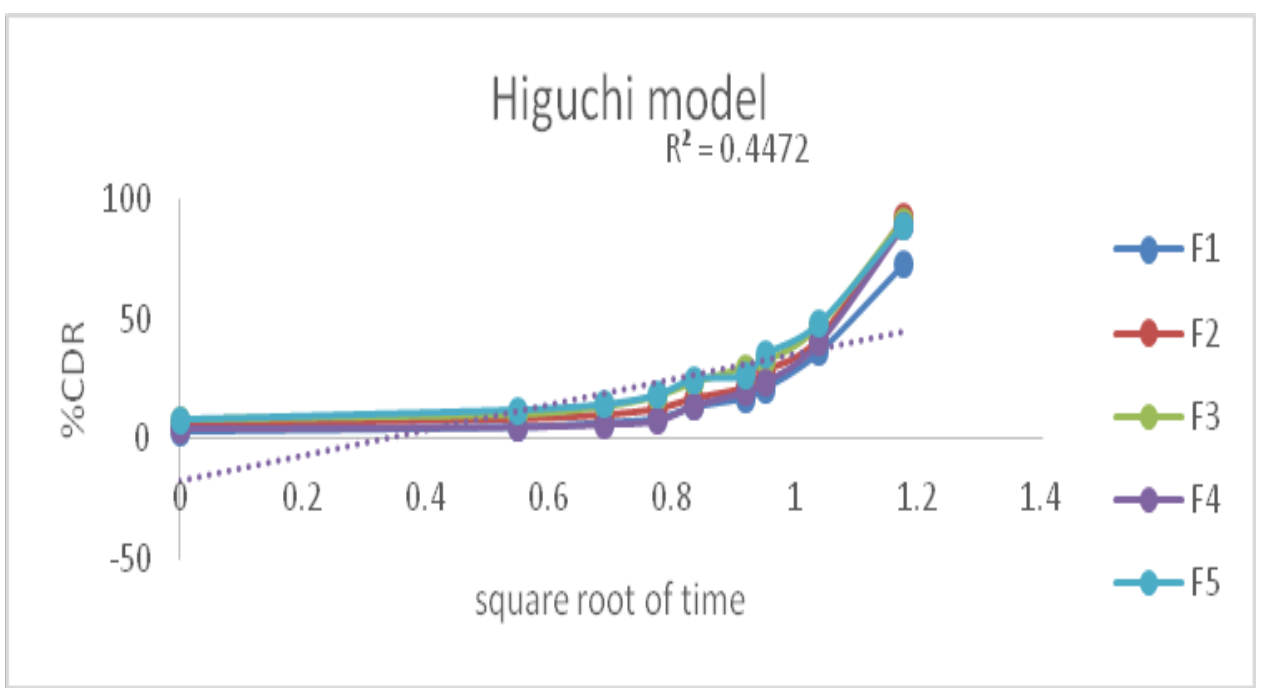

Fig No.20: Plot of percentage CDR v/s square root of time

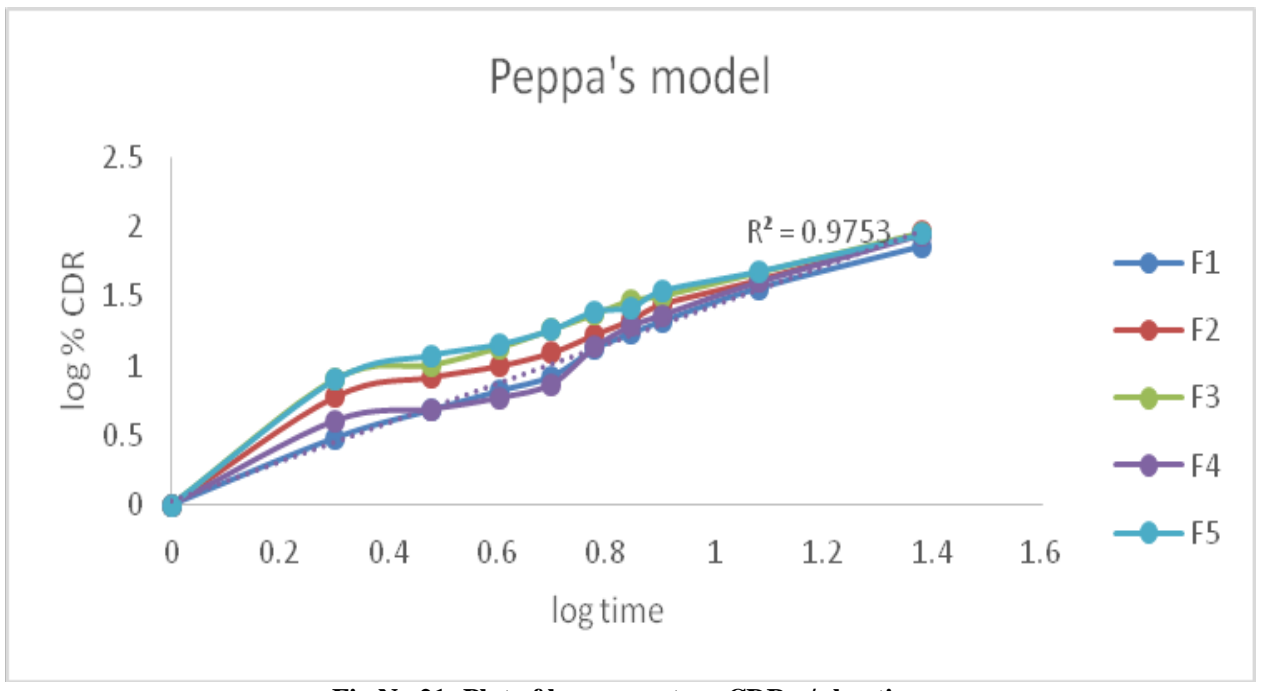

Fig No.21: Plot of log percentage CDR v/s log time

Various dissolving models were used to the in-vitro release profiles of the optimum formulation to explore the release mechanism of nanoemulsions. Zero order, first order, Higuchi, and Korsmeyer-Peppas equations were among the kinetic models used. The values of correlation-coefficient (r2) for all formulations were high enough to evaluate drug dissolving behaviour, as shown in tables 14 to 18 . Because r2 values are larger than those of other release kinetics, kinetic findings indicated that all formulations followed Zero order kinetic release. Figures 18 through 21 depicted the results.

\section{EVALUATION OF NANOEMULGEL}

\section{Physical examination}

Tab.No.19: Physical examination of various formulations of nanoemulgel

\begin{tabular}{|l|l|l|l|l|}
\hline Formulation code & Appearance & Phase Separation & Homogeneity & Consistency \\
\hline GF1 & White & None & Excellent & Good \\
\hline GF2 & White & None & Excellent & Excellent \\
\hline GF3 & White & None & Good & Excellent \\
\hline GF4 & White & None & Fair & Fair \\
\hline GF5 & White & None & Excellent & Good \\
\hline
\end{tabular}


There was no phase separation in any of the Miconazole Nitrate nanoemulgel formulations, which were white viscous preparations with a smooth and uniform appearance. Table No.19 shows the results of physical examinations. Figure No. 22 shows how to make nanoemulgel.

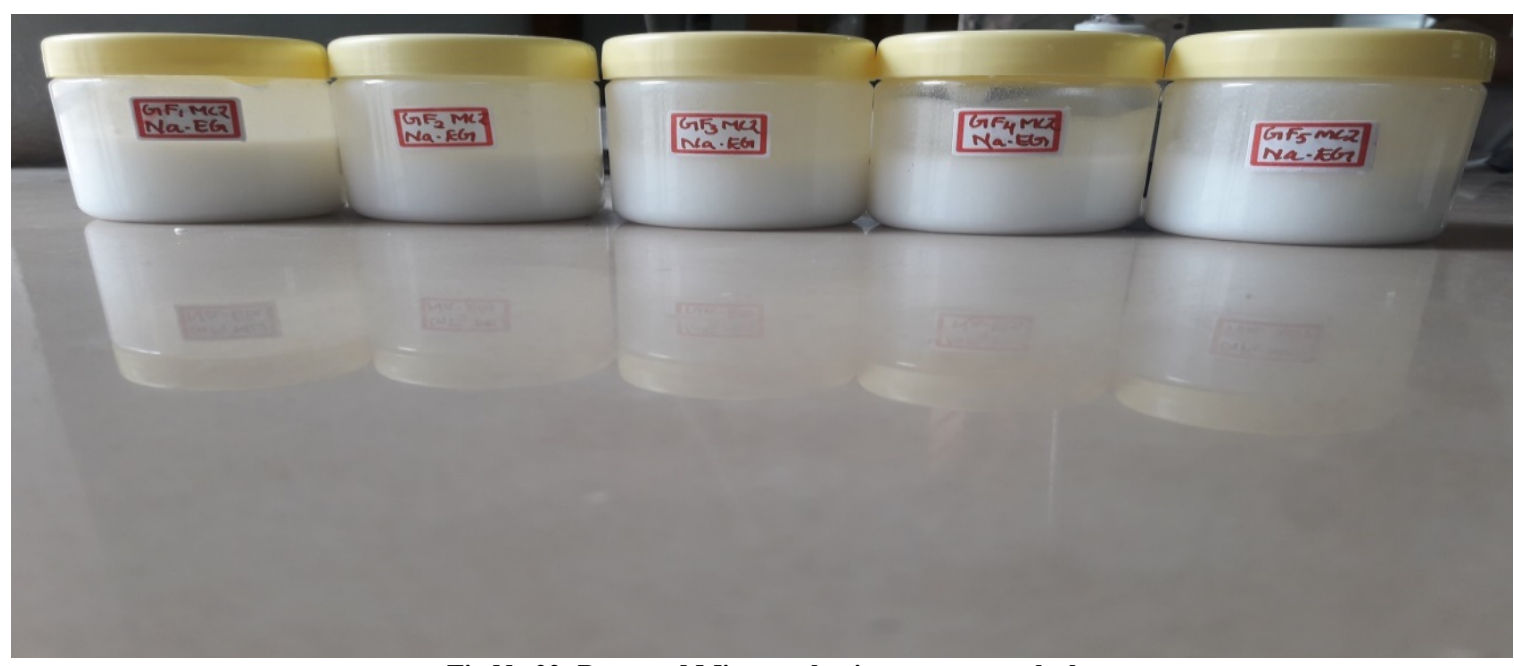

Fig.No.22: Prepared Miconazole nitrate nanoemulgel

\section{Measurement of $\mathbf{p H}$}

Tab.No.15: $\mathrm{pH}$ of various formulations of nanoemulgel

\begin{tabular}{|l|l|}
\hline Formulation code & pH \\
\hline GF1 & $6.5 \pm 0.01$ \\
\hline GF2 & $6.2 \pm 0.03$ \\
\hline GF3 & $6.1 \pm 0.10$ \\
\hline GF4 & $6.4 \pm 0.06$ \\
\hline GF5 & $6.3 \pm 0.10$ \\
\hline
\end{tabular}

All formulations had $\mathrm{pH}$ values ranging from 6.1 to 6.5 , which are deemed suitable for avoiding skin irritation when applied to the skin. Table No.15 shows the $\mathrm{pH}$ of all of the formulations.

\section{Rheological Study}

Tab.No.16: Viscosity of various formulations of nanoemulgel

\begin{tabular}{|l|l|}
\hline Formulation code & $\begin{array}{l}\text { Viscosity } \\
\text { (g/cm2) }\end{array}$ \\
\hline GF1 & 16.3 \\
\hline GF2 & 24.3 \\
\hline GF3 & 21.9 \\
\hline GF4 & 19.8 \\
\hline GF5 & 16.8 \\
\hline
\end{tabular}

The viscosities of various formulations were measured using a Brookfield viscometer at $37^{\circ} \mathrm{C}$ and $100 \mathrm{rpm}$ with spindle no. 64. Table No.21 shows the viscosities of all the formulas. The viscosity ranges from 16.3 to $24.3 \mathrm{~g} / \mathrm{cm} 2$

\section{Spreadability}

Tab.No.17: Spreadability of various formulations of nanoemulgel

\begin{tabular}{|l|l|}
\hline Formulation code & $\begin{array}{l}\text { Spreadability } \\
\text { (g cm/sec) }\end{array}$ \\
\hline GF1 & $19.25 \pm 0.12$ \\
\hline GF2 & $26.33 \pm 0.15$ \\
\hline GF3 & $24.01 \pm 0.44$ \\
\hline GF4 & $22.37 \pm 0.10$ \\
\hline GF5 & $20.31 \pm 0.19$ \\
\hline
\end{tabular}

Table No.17 displays the spreadability values. The highest spreadability was achieved by GF2 (26.33g $\mathrm{cm} / \mathrm{sec}$ ), whereas the smallest spreadability was achieved by GF1 (16.3g cm/sec).

\section{Drug Content Determination}

Tab.No.18: \% Drug content of various formulations of Nanoemulgel

\begin{tabular}{|l|l|}
\hline Formulation code & \% Drug content \\
\hline GF1 & $81.19 \pm 0.43$ \\
\hline GF2 & $94.45 \pm 0.01$ \\
\hline GF3 & $89.43 \pm 0.27$ \\
\hline GF4 & $88.18 \pm 0.14$ \\
\hline GF5 & $83.52 \pm 0.19$ \\
\hline
\end{tabular}




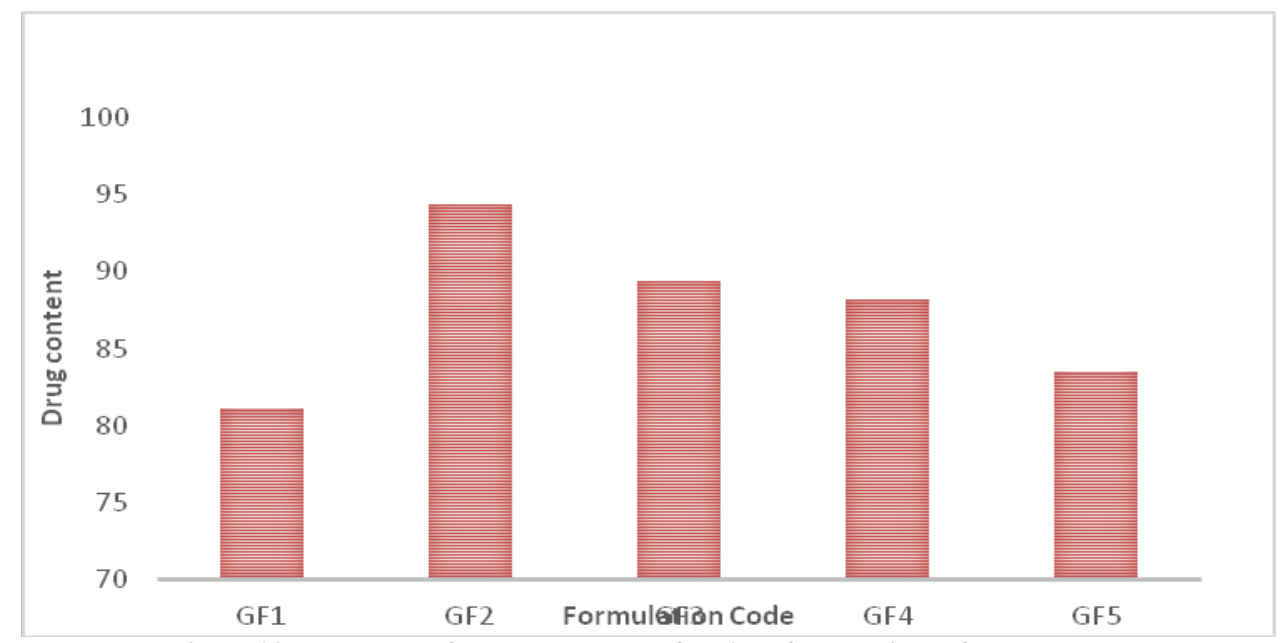

Fig.No.23: Bar graph of \% Drug content of various formulations of nanoemulgel

The drug content of nanoemulgels was determined by spectrophotometry at $272 \mathrm{~nm}$, with drug concentrations ranging from 81.19 to 94.45 percent. GF2 had the highest drug content (94.45 percent), whereas F1 had the lowest drug level (81.19 percent). Fig No.23 shows the findings.

\section{Comparison of In-vitro release study of formulations with marketed product}

Tab no.24: Comparison of In-vitro release study of GF-Formulations and Marketed product Dk gel
\begin{tabular}{|l|l|l|l|l|l|l|}
\hline \multirow{2}{*}{ Time in hr } & Percentage cumulative drug release* \\
\cline { 2 - 7 } & GF1 & GF2 & GF3 & GF4 & GF5 & Marketed product \\
\hline 1 & $14.2 \pm 0.01$ & $18.07 \pm 0.01$ & $17.55 \pm 0.03$ & $17.36 \pm 0.02$ & $22.79 \pm 0.04$ & $20.92 \pm 0.02$ \\
\hline 2 & $18.26 \pm 0.01$ & $27.26 \pm 0.02$ & $25.51 \pm 0.05$ & $23.81 \pm 0.04$ & $23.56 \pm 0.01$ & $27.5 \pm 0.03$ \\
\hline 3 & $26.22 \pm 0.04$ & $30.23 \pm 0,01$ & $29.62 \pm 0.01$ & $27.58 \pm 0.01$ & $25.8 \pm 0.02$ & $34.75 \pm 0.01$ \\
\hline 4 & $28.45 \pm 0.02$ & $38.45 \pm 0.05$ & $36.44 \pm 0.04$ & $35.72 \pm 0.02$ & $33.62 \pm 0.03$ & $38.36 \pm 0.04$ \\
\hline 5 & $34.24 \pm 0.01$ & $44.11 \pm 0.01$ & $42.11 \pm 0.01$ & $41.51 \pm 0.04$ & $40.86 \pm 0.04$ & $42.86 \pm 0.15$ \\
\hline 12 & $52.71 \pm 0.02$ & $60.56 \pm 0.02$ & $56.55 \pm 0.02$ & $54.98 \pm 0.03$ & $52.19 \pm 0.01$ & $65.5 \pm 0.04$ \\
\hline 24 & $74.05 \pm 0.01$ & $96.5 \pm 0.04$ & $92.62 \pm 0.02$ & $93.64 \pm 0.04$ & $89.57 \pm 0.01$ & $93.67 \pm 0.03$ \\
\hline
\end{tabular}

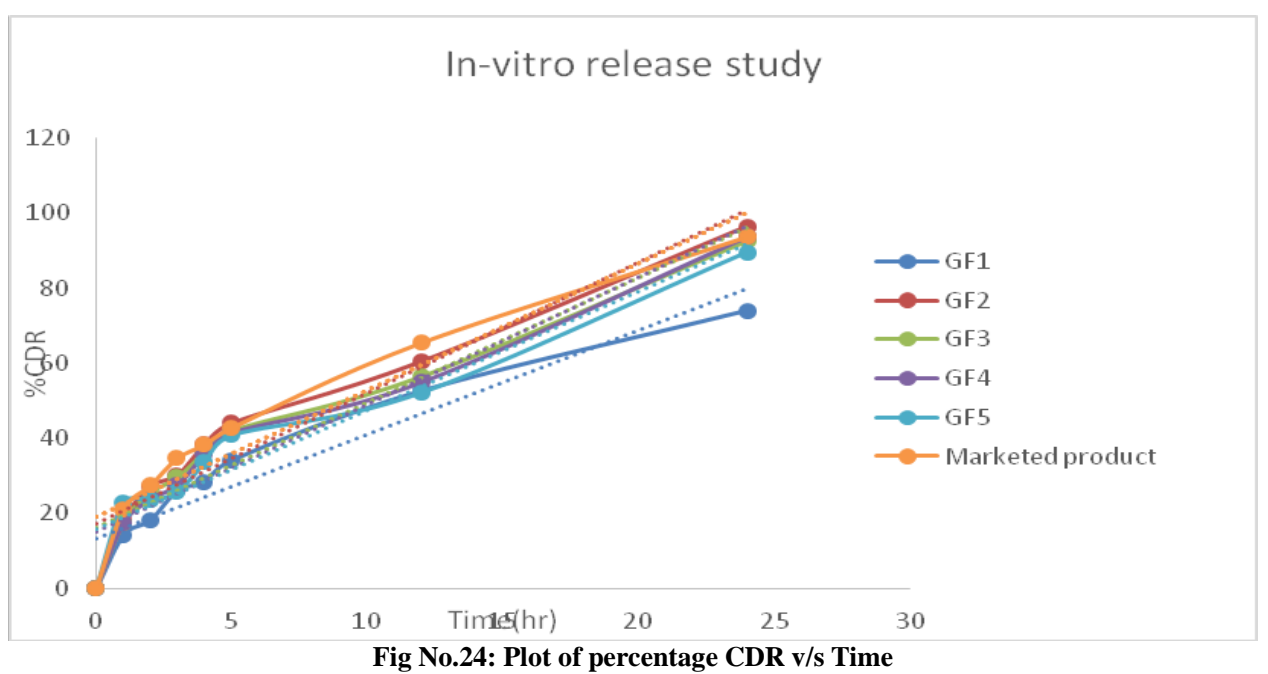

The materials were examined spectrophotometrically at $272 \mathrm{~nm}$ after being incubated for 24 hours in phosphate buffer pH 5.5 and methanol (80:20) in a Franz diffusion cell. The GF2 has a superior outcome. Table No.24 displays the results. 
Anju K P et.al. Antifungal topical nanoemulgel containing miconazole nitrate.

\section{Drug release kinetic studies}

Table no. 25: Drug release kinetics of GF1

\begin{tabular}{|l|l|l|l|l|l|l|}
\hline Time & $\begin{array}{l}\text { Log } \\
\text { time }\end{array}$ & $\begin{array}{l}\text { Square root of } \\
\text { time }\end{array}$ & $\begin{array}{l}\text { \%cumulative release } \\
\text { of GF1 }\end{array}$ & $\begin{array}{l}\text { Log \% cumulative } \\
\text { release of GF1 }\end{array}$ & $\begin{array}{l}\text { \%Cumulative } \\
\text { remaining }\end{array}$ & $\begin{array}{l}\text { Log cumulative } \\
\text { remaining }\end{array}$ \\
\hline 0 & 0 & 0 & 0 & 0 & 100 & 2 \\
\hline 1 & 0 & 0 & 14.2 & 1.152288 & 85.8 & 1.933487 \\
\hline 2 & 0.30103 & 0.548662 & 18.26 & 1.261501 & 81.74 & 1.912435 \\
\hline 3 & 0.477121 & 0.69074 & 26.22 & 1.418633 & 73.78 & 1.867939 \\
\hline 4 & 0.60206 & 0.775925 & 28.45 & 1.454082 & 71.55 & 1.85461 \\
\hline 5 & 0.69897 & 0.836044 & 34.24 & 1.534534 & 65.76 & 1.817962 \\
\hline 12 & 1.079181 & 1.038836 & 52.71 & 1.721893 & 47.29 & 1.674769 \\
\hline 24 & 1.380211 & 1.174824 & 74.05 & 1.869525 & 25.95 & 1.414137 \\
\hline
\end{tabular}

Table no. 26: Drug release kinetics of GF2

\begin{tabular}{|l|l|l|l|l|l|l|}
\hline Time & $\begin{array}{l}\text { Log } \\
\text { time }\end{array}$ & $\begin{array}{l}\text { Square root of } \\
\text { time }\end{array}$ & $\begin{array}{l}\text { \%cumulative release } \\
\text { of GF2 }\end{array}$ & $\begin{array}{l}\text { Log \% cumulative } \\
\text { release of GF2 }\end{array}$ & $\begin{array}{l}\text { \%Cumulative } \\
\text { remaining }\end{array}$ & $\begin{array}{l}\text { Log cumulative } \\
\text { remaining }\end{array}$ \\
\hline 0 & 0 & 0 & 0 & 0 & 100 & 2 \\
\hline 1 & 0 & 0 & 18.07 & 1.256958 & 81.93 & 1.913443 \\
\hline 2 & 0.30103 & 0.548662 & 27.26 & 1.435526 & 72.74 & 1.861773 \\
\hline 3 & 0.477121 & 0.69074 & 30.23 & 1.480438 & 69.77 & 1.843669 \\
\hline 4 & 0.60206 & 0.775925 & 38.45 & 1.584896 & 61.55 & 1.789228 \\
\hline 5 & 0.69897 & 0.836044 & 44.11 & 1.644537 & 55.89 & 1.747334 \\
\hline 12 & 1.079181 & 1.038836 & 60.56 & 1.782186 & 39.44 & 1.595937 \\
\hline 24 & 1.380211 & 1.174824 & 96.5 & 1.984527 & 3.5 & 0.544068 \\
\hline
\end{tabular}

Table no. 27: Drug release kinetics of GF3

\begin{tabular}{|l|l|l|l|l|l|l|}
\hline Time & $\begin{array}{l}\text { Log } \\
\text { time }\end{array}$ & $\begin{array}{l}\text { Square root of } \\
\text { time }\end{array}$ & $\begin{array}{l}\text { \%cumulative release } \\
\text { of GF3 }\end{array}$ & $\begin{array}{l}\text { Log \% cumulative } \\
\text { release of GF3 }\end{array}$ & $\begin{array}{l}\text { \%Cumulative } \\
\text { remaining }\end{array}$ & $\begin{array}{l}\text { Log cumulative } \\
\text { remaining }\end{array}$ \\
\hline 0 & 0 & 0 & 0 & 0 & 100 & 2 \\
\hline 1 & 0 & 0 & 17.55 & 1.244277 & 82.45 & 1.916191 \\
\hline 2 & 0.30103 & 0.548662 & 25.51 & 1.40671 & 74.49 & 1.872098 \\
\hline 3 & 0.477121 & 0.69074 & 29.62 & 1.471585 & 70.38 & 1.847449 \\
\hline 4 & 0.60206 & 0.775925 & 36.44 & 1.561578 & 63.56 & 1.803184 \\
\hline 5 & 0.69897 & 0.836044 & 42.11 & 1.624385 & 57.89 & 1.762604 \\
\hline 12 & 1.079181 & 1.038836 & 56.55 & 1.752433 & 43.45 & 1.63799 \\
\hline 24 & 1.380211 & 1.174824 & 92.62 & 1.966705 & 7.38 & 0.868056 \\
\hline
\end{tabular}

Table no. 28: Drug release kinetics of GF4

\begin{tabular}{|l|l|l|l|l|l|l|}
\hline Time & $\begin{array}{l}\text { Log } \\
\text { time }\end{array}$ & $\begin{array}{l}\text { Square root of } \\
\text { time }\end{array}$ & $\begin{array}{l}\text { \%cumulative release } \\
\text { of GF4 }\end{array}$ & $\begin{array}{l}\text { Log \% cumulative } \\
\text { release of GF4 }\end{array}$ & $\begin{array}{l}\text { \%Cumulative } \\
\text { remaining }\end{array}$ & $\begin{array}{l}\text { Log cumulative } \\
\text { remaining }\end{array}$ \\
\hline 0 & 0 & 0 & 0 & 0 & 100 & 2 \\
\hline 1 & 0 & 0 & 17.36 & 1.23955 & 82.64 & 1.91719 \\
\hline 2 & 0.30103 & 0.548662 & 23.81 & 1.376759 & 76.19 & 1.881898 \\
\hline 3 & 0.477121 & 0.69074 & 27.58 & 1.440594 & 72.42 & 1.859859 \\
\hline 4 & 0.60206 & 0.775925 & 35.72 & 1.552911 & 64.28 & 1.808076 \\
\hline 5 & 0.69897 & 0.836044 & 41.51 & 1.618153 & 58.49 & 1.767082 \\
\hline 12 & 1.079181 & 1.038836 & 54.98 & 1.740205 & 45.02 & 1.653405 \\
\hline 24 & 1.380211 & 1.174824 & 93.64 & 1.971461 & 6.36 & 0.803457 \\
\hline
\end{tabular}

Table no. 29: Drug release kinetics of GF5

\begin{tabular}{|l|l|l|l|l|l|l|}
\hline Time & $\begin{array}{l}\text { Log } \\
\text { time }\end{array}$ & $\begin{array}{l}\text { Square root of } \\
\text { time }\end{array}$ & $\begin{array}{l}\text { \%cumulative release } \\
\text { of GF5 }\end{array}$ & $\begin{array}{l}\text { Log \% cumulative } \\
\text { release of GF5 }\end{array}$ & $\begin{array}{l}\text { \%Cumulative } \\
\text { remaining }\end{array}$ & $\begin{array}{l}\text { Log cumulative } \\
\text { remaining }\end{array}$ \\
\hline 0 & 0 & 0 & 0 & 0 & 100 & 2 \\
\hline 1 & 0 & 0 & 22.79 & 1.357744 & 77.21 & 1.887674 \\
\hline 2 & 0.30103 & 0.548662 & 23.56 & 1.372175 & 76.44 & 1.883321 \\
\hline 3 & 0.477121 & 0.69074 & 25.8 & 1.41162 & 74.2 & 1.870404 \\
\hline 4 & 0.60206 & 0.775925 & 33.62 & 1.526598 & 66.38 & 1.822037 \\
\hline 5 & 0.69897 & 0.836044 & 40.86 & 1.611298 & 59.14 & 1.771881 \\
\hline 12 & 1.079181 & 1.038836 & 52.19 & 1.717587 & 47.81 & 1.679519 \\
\hline 24 & 1.380211 & 1.174824 & 89.57 & 1.952163 & 10.43 & 1.018284 \\
\hline
\end{tabular}

Table no. 30:Drug release kinetics of Marketed Product formulation

\begin{tabular}{|l|l|l|l|l|l|l|}
\hline Time & $\begin{array}{l}\text { Log } \\
\text { time }\end{array}$ & $\begin{array}{l}\text { Square root } \\
\text { of time }\end{array}$ & $\begin{array}{l}\text { \%cumulative release } \\
\text { of marketed product }\end{array}$ & $\begin{array}{l}\text { Log \% cumulative release } \\
\text { of marketed product }\end{array}$ & $\begin{array}{l}\text { \%Cumulative } \\
\text { remaining }\end{array}$ & $\begin{array}{l}\text { Log cumulative } \\
\text { remaining }\end{array}$ \\
\hline 0 & 0 & 0 & 0 & 0 & 100 & 2 \\
\hline 1 & 0 & 0 & 20.92 & 1.320562 & 79.08 & 1.898067 \\
\hline 2 & 0.30103 & 0.548662 & 27.5 & 1.439333 & 72.5 & 1.860338 \\
\hline 3 & 0.477121 & 0.69074 & 34.75 & 1.540955 & 65.25 & 1.814581 \\
\hline 4 & 0.60206 & 0.775925 & 38.36 & 1.583879 & 61.64 & 1.789863 \\
\hline 5 & 0.69897 & 0.836044 & 42.86 & 1.632052 & 57.14 & 1.75694 \\
\hline 12 & 1.079181 & 1.038836 & 65.5 & 1.816241 & 34.5 & 1.537819 \\
\hline 24 & 1.380211 & 1.174824 & 93.67 & 1.971601 & 6.33 & 0.801404 \\
\hline
\end{tabular}




\section{Zero order release:}

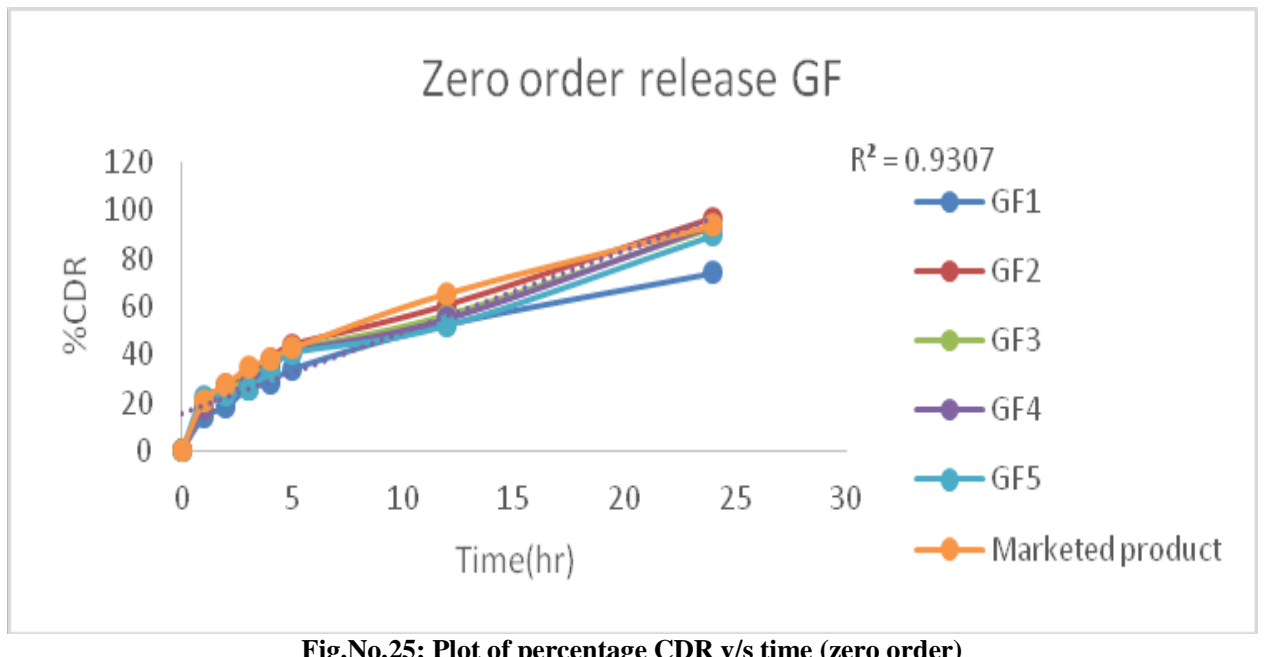

Fig.No.25: Plot of percentage CDR v/s time (zero order)

\section{First order release:}

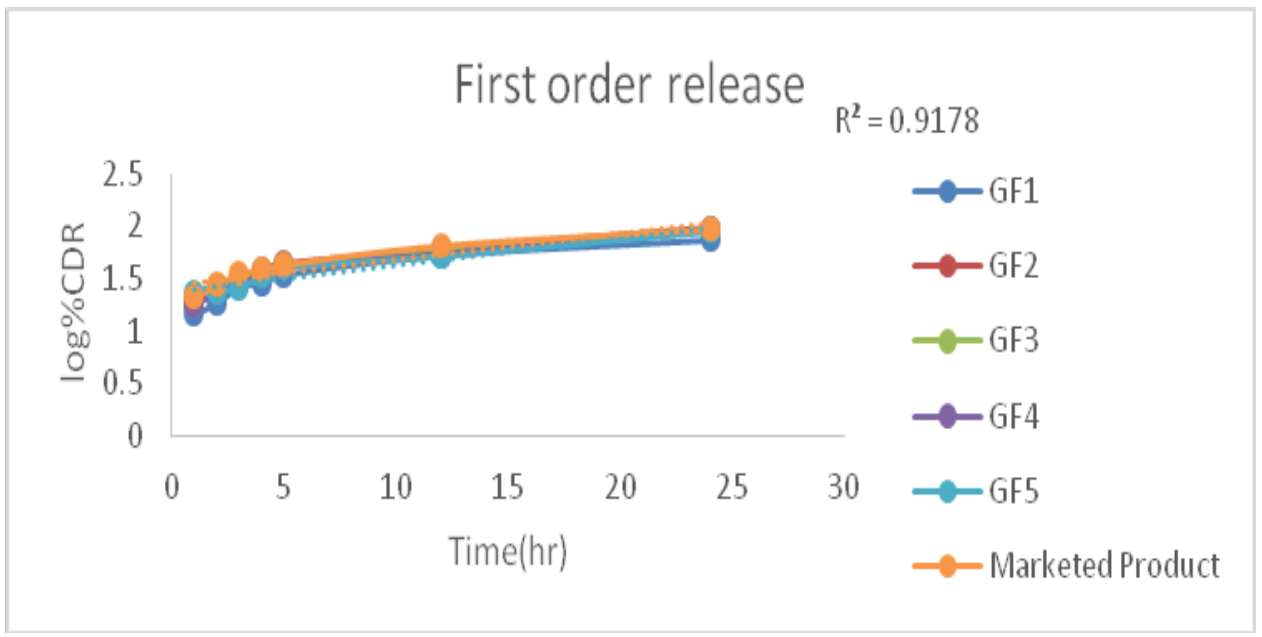

Fig.No.26: Plot of log percentage CDR v/s time (first order)

\section{Peppa's model:}

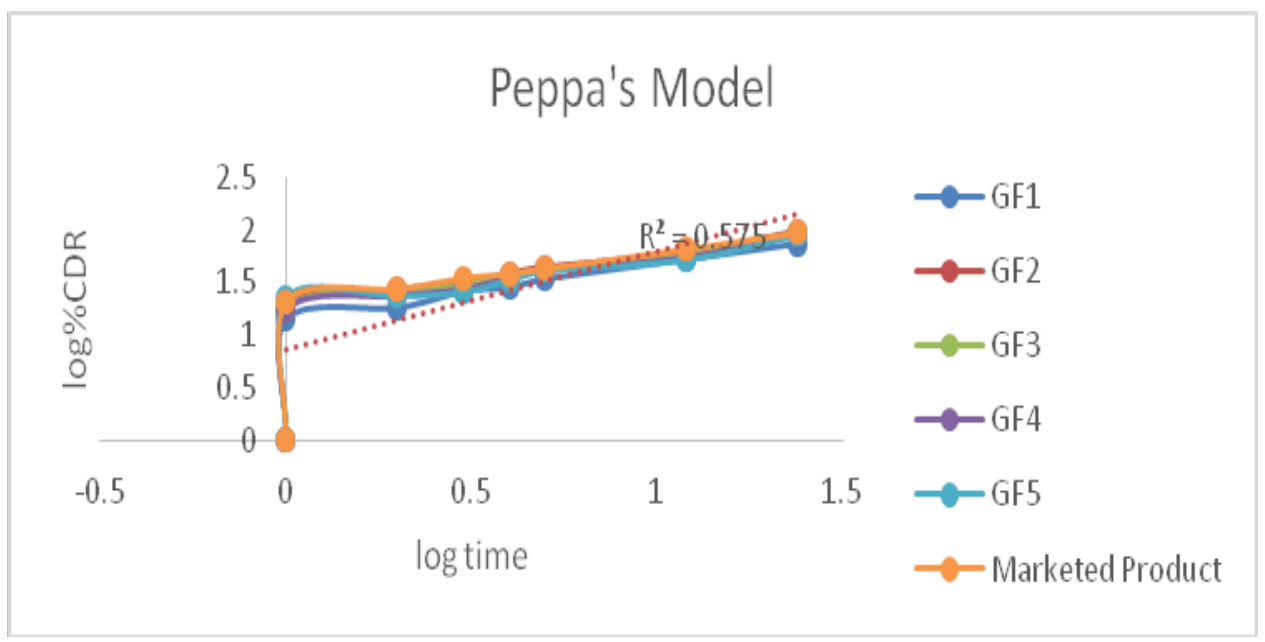

Fig.No.27: Plot of log percentage CDR v/s log time (Peppa's model) 


\section{Higuchi's model:}

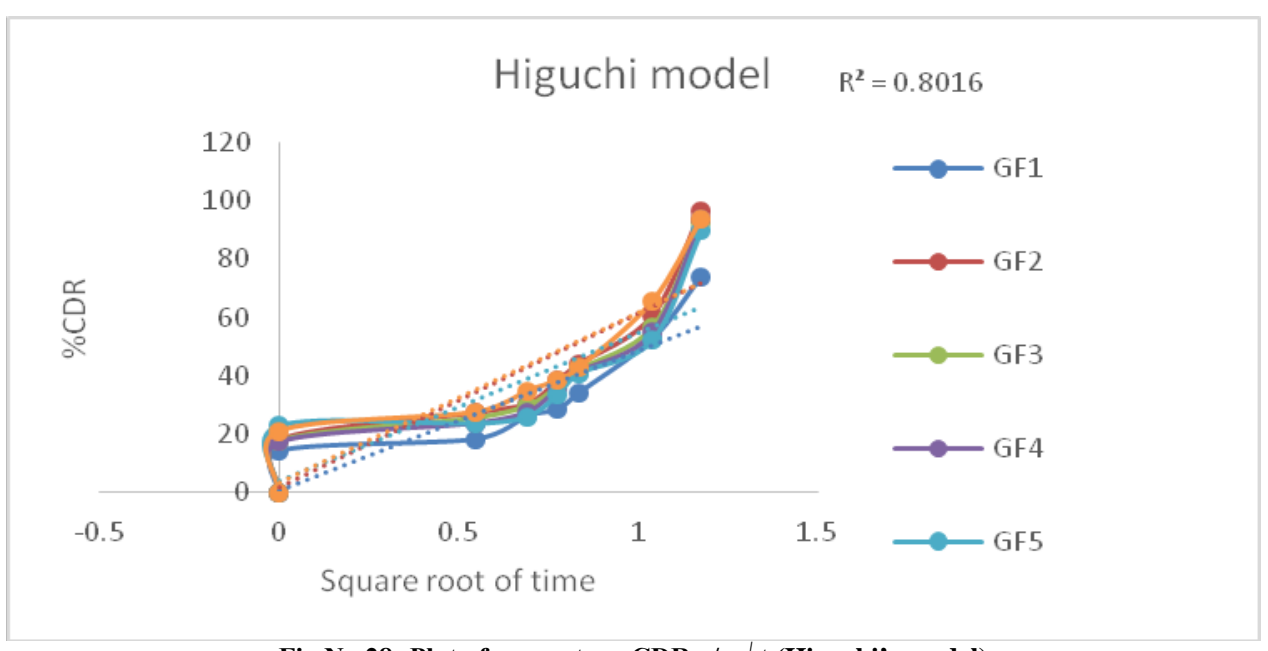

Fig.No.28: Plot of percentage CDR v/s $\sqrt{ }$ t (Higuchi’s model)

Various dissolving models were used to the in-vitro release profiles of formulations to explore the release mechanism of nanoemulgels. Zero order, first order, Higuchi, and Korsmeyer-Peppas equations were among the kinetic models used. The values of correlation-coefficient (r2) for all formulations were high enough to evaluate drug dissolving behaviour, as shown in table no.20. Because r2 values are larger than those of other release kinetics, kinetic findings indicated that all formulations followed Zero order kinetic release. Tables 25 to 30 and figures 25 to 28 illustrate the results.

\section{Accelerated stability studies}

Tab.No.31: stability study of GF2

\begin{tabular}{|c|c|c|c|}
\hline \multirow[t]{2}{*}{$\begin{array}{l}\text { Evaluation } \\
\text { Parameters }\end{array}$} & \multicolumn{3}{|c|}{$\begin{array}{l}\text { Time (days) } \\
\text { Accelerated condition at } 40 \pm 2^{\circ} \mathrm{C} \text { and } 75 \pm 5 \% \\
\text { RH }\end{array}$} \\
\hline & At 0 days & After 30 days & After 60 days \\
\hline Colour & White & White & White \\
\hline $\begin{array}{l}\text { Phase } \\
\text { separation }\end{array}$ & None & None & None \\
\hline Drug content & 94.45 & 90.39 & 90.3 \\
\hline
\end{tabular}

\section{In-vitro drug release study}

Tab.No.32: In-vitro drug release of GF2 during stability study

\begin{tabular}{|l|l|l|l|}
\hline \multirow{2}{*}{$\begin{array}{l}\text { Time } \\
\text { (hrs) }\end{array}$} & \multicolumn{3}{|l|}{$\begin{array}{l}\text { \% CDR } \\
\text { Accelerated condition at } \mathbf{4 0} \pm \mathbf{2}^{\mathbf{}} \mathbf{C} \text { and } \mathbf{7 5} \pm \mathbf{5} \% \mathbf{R H}\end{array}$} \\
\cline { 2 - 4 } & At 0 days & After 30th day & After $\mathbf{6 0 t h}$ day \\
\hline 0 & 0 & 0 & 0 \\
\hline 1 & 18.07 & 17.98 & 17.62 \\
\hline 2 & 27.26 & 26.26 & 26.02 \\
\hline 3 & 30.23 & 30.03 & 29.96 \\
\hline 4 & 38.45 & 37.72 & 37.04 \\
\hline 5 & 44.11 & 43.94 & 43.72 \\
\hline 12 & 60.56 & 60.01 & 59.98 \\
\hline 24 & 96.5 & 95.94 & 95.88 \\
\hline
\end{tabular}

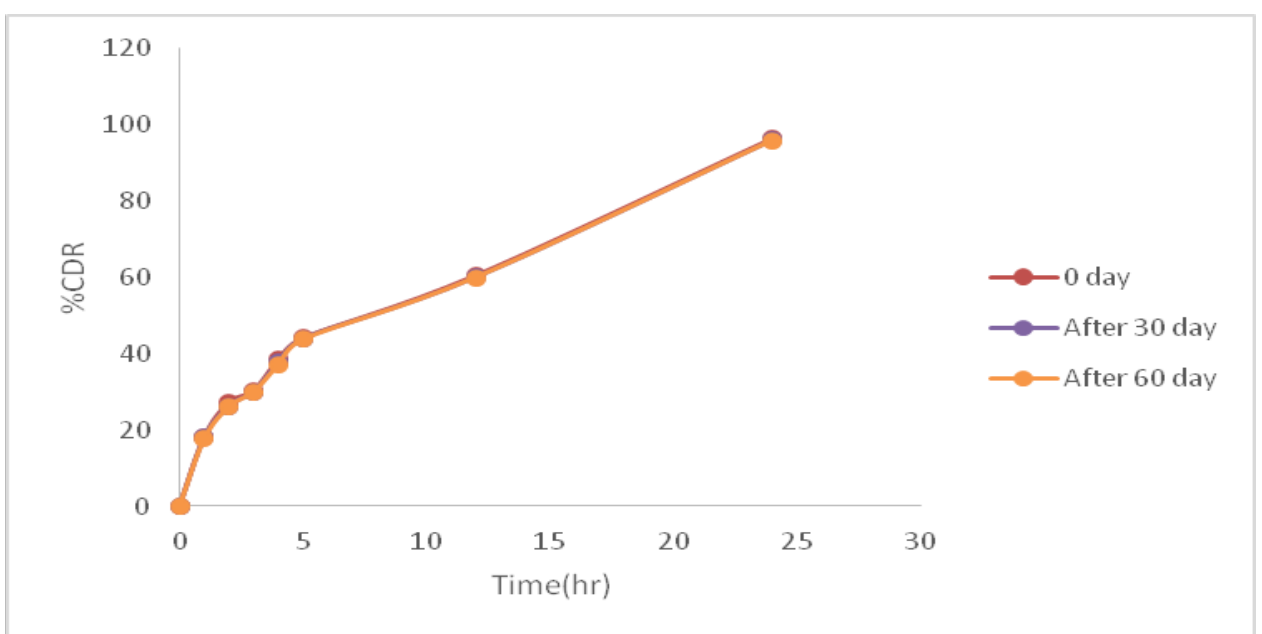

Fig no.29: In-Vitro release of GF2 during stability Study. 
Stability tests were performed on chosen formulations F2 in accordance with ICH standards Q1C. The best formulas were kept for two months in sealed aluminium tubes at 40 degrees Celsius and $75 \%$ relative humidity. During the research, there was no change in colour, phase separation, or drug content. After two months, the percentage drug release in F2 after 24 hours was 95.88 percent, suggesting no major alterations and that nanoemulgels are stable in storage. The results are shown in table Nos. 31 and 32, as well as picture No. 29.

\section{SUMMARY AND CONCLUSION}

The goal of this research was to design and develop a Miconazole nitrate nanoemulgel to treat fungal infections on the skin. Miconazole nitrate preformulation experiments were found to be within the published literature limitations. FTIR tests indicated no chemical interactions between the drug, the polymer, and the excipients, indicating that the drug is stable in the formulation. High-pressure homogenization was used to create nanoemulsions. Physical tests of the produced nanoemulsions revealed that they were milky white with a smooth and homogeneous appearance and good consistency, indicating that the nanoemulsions were stable. The $\mathrm{pH}$, drug content, and centrifugation stability tests all reported back within acceptable limits. The drug content of all formulations was determined, and the F1, F2, F3, F4, and F5 values were $79.13,91.56,88.55,86.12$, and 81.46 percent, respectively. The formulations were determined to be stable and homogenous, and there was no coalescence in the nanoemulsion, according to globule size determination and zeta potential tests. The size distribution of globules varies from 7.9 to 41.6 d.nm. Nanoemulsion Formulation F2 was judged to be excellent based on assessment factors. The nanoemulgels were made by mixing o/w emulsion with carbopol 934, and all of the results were consistent and stable. The $\mathrm{pH}$, viscosity, and spreadability tests were completed, and the findings were consistent and reproducible. The drug content of the formulations was analyzed, and it was determined that the drug content GF2 is satisfactory. The Franz diffusion cell was used to conduct in-vitro release experiments on all formulations for 24 hours. The greatest release was 96.5 percent for GF2, while the highest release was 93.69 percent for the marketed product. All formulations were subjected to kinetic drug release experiments, and all of them followed zero order kinetics. For the best formulations GF2, stability experiments were carried out for two months, and the findings of appearance, phase separation, and drug content were all within the literature limitations. In-vitro drug release tests revealed no significant alterations, leading to the conclusion that all formulations were stable during storage. The Nanoemulgels were shown to be stable and to release Miconazole nitrate effectively. The current investigation shown that carbopol 934 may be used to successfully produce Miconazole Nitrate nanoemulgel formulations. Nanoemulgels appear to be a reliable approach for topical administration of hydrophobic medicines in water soluble gel bases.

\section{ACKNOWLEDGMENT}

I pay my deep sense of gratitude to Management of Srinivas College of Pharmacy and Vision Group for Science and Technology, Govt of Karnataka.

\section{Conflict of Interest: None}

\section{Source of Funding: None}

\section{Ethical Approval: Approved}

\section{REFERENCES}

1. Hira C,Manisha P,Lipika AC,Nagashekhara M.Recent Update On Nanoemulgel As Topical Drug Delivery System.Journal of Pharmaceutical Sciences 2017;106(7):173651.

2. Shanker DS.Ishak RA,Ghoneim A,Elhuoni MA. Nanoemulsion: A Review on Mechanisms For the Transdermal Delivery 
of Hydrophobic and Hydrophilic Drugs. Scientia Pharmaceutica 2019;87(3):17-50.

3. Raj KM,Soni GC,Mishra RP. A Review Article: On Nanoemulsion.World Journal Of Pharmacy and Pharmaceutical Sciences 2014;3(9):258-74.

4. Asish D, Reha C, Om S. Emulgels: a novel topical drug delivery system. Pharm and Bio Eval 2015; 2 (4): 64-75.

5. Haneefa MKP, Prasad GM, Chandini N. Formulation and Evaluation of Herbal Emulgel of Pothos scandens Linn for Burn Wound Healing Activity. J. Pharm. Sci \& Res 2014; 6(2): 63-67.

6. Davinder K, Jasbir S, Mamta A, Virender K. Emulgel-Novel topical drug delivery system-a comprehensive review. IJPSR2016; 7(12): 4733-42.

7. Vaishali A, Praveen K, Rajni S. Emulgels: A review for topical drug delivery of hydrophobic drugs. Int J Pharm Profl's Res. 2015; 6 (3): 1256-63.

8. Baibhav J, Gurpreet S, Rana AC, Seema S, Vikas S. Emulgel: A Comprehensive review on the recent advances in topical drug delivery. Int Res J Pharm 2011; 2(11): 6670.

9. Remington: The Science and Practice of Pharmacy, 20th Edition, Edited by Alfonso R Gennaro Published by Lippincot Williams and Wilkins, Philadelphia 2000; 1114, 1147, 1200

10. Nandini G, Sirisha B. Review on topical Gellified emulsion: Superior for hydrophobic drugs.Int J Pharm and Anal res 2015; 4(3): 276-81.

11. Rahul RP, Patel KR, Patel MR. Formulation and Characterization of Microemulsion based Gel of Antifungal Drug. Pharm Tutor 2014; 2(2): 79-89.

12. Panwar AS, Upadhyay N, Bairagi M, Gujar S, Darwhekar GN, Jain DK. Emulgel: A review. J Pharm and Life Sci 2011; 1 (3): 333-43.

13. Vikas S, Seema S, Baibhav J and Rana AC. Emulgel: A new platform for topical drug delivery. Int J Pharma Bio Sci 2012; 3(1): 485-98.

14. Arpan AS, Kamdar K, Rushabh S, Rajesh AK. Emulgel: A Topical Preparation for Hydrophobic Drugs. Ph Tech Med 2013; 2(5): 370-76.

15. Hardenia A, Sonali J, Sanjay J. Emulgel: An emergent tool in topical drug delivery. Int J Pharm Sci Res 2014; 5 (5):1653-60.
16. Rachit K, Saini S, Seth N, Rana AC. Emulgels: A surrogate approach for topically used hydrophobic drugs. Int J Pharm and Bio Sci 2011; 1(3): 117-28.

17. Supriya U, Seema C, Preeti K. Emulgel: A Boon for Dermatological Diseases. Int J Pharma Res Al Sci 2014; 3(4): 1-9.

18. Meenakshi D. Emulgel: A Novel Approach to Topical drug delivery. Int J Pharm Bio Sci 2013; 4(1): 847 - 56.

19. Kute SB, Saudagar RB. Emulsified gel A Novel approach for delivery of hydrophobic drugs: An overview. J Adv Pharm Edu \& Res 2013; 3(4): 368-76.

20. Madhavi LS, Sridevi G. Role of Polymers as Gelling Agents in the Formulation of Emulgels. IMed Pub J 2016; 1(10): 1-8.

21. Dhruti PM, Hemendrasinh JR, Dhiren PS, Chainesh NS. A Review on Microemulsion Based Gel: A Recent Approach for Topical Drug Delivery System. Res J Pharm and Tech 2015; 8 (2): 118-26.

22. Robert AF. Overview of medically important antifungal Azole derivatives. Cli micr rev 1988; 1(2): 187-217.

23. Bharat P, Atul K, Ajay C. Formulation and Evaluation Gel Containing Miconazole Nitrate an Antifungal Agent. Int J Pharm Res and Rev 2013; 2(6): 18-28.

24. Sevgi, Sedef EM, Buket A. New Formulation Strategies in Topical Antifungal Therapy. J Cos, Derm Scie and Appl 2013; 3, 56-65.

25. Yassin GE. Formulation and evaluation of optimized Clotrimazole emulgel formulations. Bri J Pharm Res 2014;4(9): 1014-30.

26. Ankur J, Surya PG, Yashwant G, Hemant $\mathrm{K}$, Sanjay J. Development and characterization of ketoconazole emulgel for topical drug delivery. Der Pharmacia Sinica 2010; 1(3): 221-31.

27. Piyusha D, Ankur J, Naveen V, Hemant K, Sanjay J. Gellified emulsion for sustain delivery of Itraconazole for topical fungal diseases. Intl J Pharm and Pharm Scie 2010; 2(1): 104-12.

28. Kaushal RS, Ganesh DB. Formulation, development and In-vitro evaluation of terbinafine hydrochloride emulgel for topical fungal infection. Int J Pharm Sci Rev Res 2013; 21(2): 168-73.

29. Priya RM, Sellakumar V, Natarajan R and Mohan K. Formulation and In-vitro Evaluation of Ciprofloxacin Loaded Topical 
Emulgel. Int J pharm chem scie 2012; 1(1): 238-42.

30. Monica R, Girish S, Sheetal A, Manmeet K. Development and optimization of metranidazole emulgel. J Pharm 2013; 1-9.

31. Bhanu PV, Shanmugam P, Lakshmi PK. Development and Optimization of novel diclofenac emulgel for topical delivery. Int J Comp pharm 2011; 2(9): 2-4.

32. Vikas S, Saini S, Rana AC, Singh G. Development and evaluation of topical emulgel of lornoxicam using different polymer bases. Int Pharm Sci 2012; 2(3): 36-44.

33. Rachit K, Deepinder K, Nirmatha S, Seema S. Formulation and evaluation of mefenamic acid emulgel for topical delivery. Saudi Pharma J 2012; 20: 63-67.

34. Khaled MH, Shatha MR, Muna M A, Samia MS, Usama AF. Ketoprofen emulgel: Preparation, characterization and pharmacodynamic evaluation. Int J Pharm Rev Res 2013; 20(2): 306-10.

35. Mohamed IM. Optimization of chlorphenesin emulgel formulation. The AAPS J 2004; 6(3): 1-7.

36. Sravan KV, Maheshwari PV, Navya M, Sharath CR, Shivakumar, Gowda DV. Calcipotriol delivery into the skin as emulgelfor effective permeation. Saudi Pharm J 2014; 22: 591-99.

37. Dignesh MK, Ashish DM, Dinesh RS. Formulation design and evaluation of piroxicam emulgel. Int $\mathrm{J}$ Pharm Tech Res 2012; 4(3): 1332-44.

38. Anwar E, Ramdon D, Harmitha. Formulation and evaluation of gel and emulgel of chili extract (Capsicum Frutescens L.) as topical dosage forms. Int $\mathrm{J}$ Pharm and Pharmaceutical Scie2014; 6(3): 13-16.

39. Janki P, Trivedi J, Sunita C. Formulation and evaluation of diacerein emulgel for psoriatic arthritis.Int J pharm Res and Bioscie 2014; 3(2): 625-38.

40. YehiaI K, Abheer HJ, Entidhar JM. Preparation and evaluation of physical and rheology study of clotrimazole emulgel. Iraqui J Pharm Sci 2011; 20(2):6-10.

41. Bhatt P, Gnanaranjan P and Preeti K. Development and characterization of salicylic acid emulgel for topial delivery by using different gelling agents. Int $\mathrm{J}$ Uni Pharm Bio Sci 2013 Sept-Oct; 2(5):374-86.
42. Lubna AS, Hala TS and Yehia IK. An investigation release and rheological properties of miconazole nitrate from emulgel. Iraqui J Pharm Sci 2009; 18(2):2631.

43. Vivek PC, Jitendra P, Kaushik P, Sanjay N. Preparation and evaluation of methyl salicylate counter irritant emulgel of mefenamic acid. Int $\mathrm{J}$ Pharma Med Res 2013; 1(1):27-38.

44. Hyndavi N, Swetha $\mathrm{K}$ and Lohita M. Formulation and evaluation of aceclofenaclycopene transemulgel. World J Pharma Res 2013; 2(4):1036-45.

45. Joshi B, Singh G, Rana AC and Saini S. Development and characterization of clarithromycin emulgel for topical delivery. Int J Drug Dev Res 2012 July Sep; 4(3):310-23.

46. Naresh KT, Prathiba B, Sheefali $M$ and Rekha R. Formulation and characterization of benzoyl peroxide gellified emulsion. Sci Pharm 2012; 80(1):1045-60.

47. Wu SX, Shen YN, Yan N, Guo NR, Liu LL, Yanq JQ. Experimental and clinical investigation on oxiconazole. Chin Med J 1989; 102(8): 644-46.

48. Jeqasothy BV, Pakes GE. Oxiconazole nitrate: pharmacology, efficacy, and safety of a new imidazole antifungal agent. Clin Ther 1991; 13(1): 126-41.

49. http://miconazole nitrate drug monograph for professionals drugs .com.htm.

50. Achhrish G, Shaweta S, Navpreet K, Anupam S. Pharmacokinetic data and solubility profile of antifugal drugs. Int $\mathrm{J}$ Pharm Prof 2011; 2(2): 256-65.

51. Rowe RC, Sheskey PJ, Owen SC. Handbook of Pharmaceutical excipients: 111-923.

52. Gupta GD, Gound RS. Release rate of nimesulide from different gelling agents. Ind J Pharm Sci 1999:61(1):229-34.

53. Indian Pharmacopeia, Govt. of India Ministry of Health \& family welfare. Published by controller of publication Delhi 1996; 2:298-301.

54. British Pharmacopoeia, MHRA, British pharmacopoeial commission office, Vol.3. London: The stationary office; 2007.

55. Abeer K, Soha I. Formulation and evaluation of oxiconazole nitrate mucoadhesive Nanoemulsion based gel for treatment of fungal vaginal infection. Int $\mathrm{J}$ of Pharm and Pharma Sci 2016; 8(3): 33-40. 
56. Bhruthika P, Kesha D,Vijeyendra SM. Formulation development and evaluation of microemulsion based hydrogel of econazole nitrate. Int J Pharm Sci 2014 Apr-Jun; 5(2):86-107.

57. Snehal PM, Kiran AW and Manish SK. Formulation, development and evaluation of Indomethacin emulgel. Der Pharmacia Sinica 2013; 4(5):31-45.

58. Bhautik K, Dipti, Dinal P, Snehal P, Chintan A, Nirmal S, Kartik P, Chainesh S.
Formulation and Evaluation of Spironolactone Loaded Emulgel for Topical Application. J Pharm Sci Bio scie Res. 2018; 6(5): 740-52.

How to cite this article: Anju K P, Shripathy D, Shabaraya A R. Antifungal topical nanoemulgel containing miconazole nitrate. Int J Health Sci Res. 2021; 11(11): 208-229. DOI: https://doi. org/10.52403/ijhsr.20211127 